$11-2008$

\title{
Image Analyses of Two Crustacean Exoskeletons and Implications of the Exoskeletal Microstructure on the Mechanical Behavior
}

\author{
Liang Cheng \\ University of Delaware \\ Liyun Wang \\ University of Delaware \\ Anette M. Karlsson \\ Cleveland State University, a.karlsson@csuohio.edu
}

Follow this and additional works at: https://engagedscholarship.csuohio.edu/enme_facpub

Part of the Mechanical Engineering Commons

How does access to this work benefit you? Let us know!

\section{Original Citation}

Cheng, Liang., and Liyun Wang, et al. (2008). Image Analyses of Two Crustacean Exoskeletons and Implications of the Exoskeletal Microstructure on the Mechanical Behavior. Journal of Materials Research, 23(11), 2854-2872.

This Article is brought to you for free and open access by the Mechanical Engineering Department at EngagedScholarship@CSU. It has been accepted for inclusion in Mechanical Engineering Faculty Publications by an authorized administrator of EngagedScholarship@CSU. For more information, please contact library.es@csuohio.edu. 


\title{
Image analyses of two crustacean exoskeletons and implications of the exoskeletal microstructure on the mechanical behavior
}

\author{
Liang Cheng, Liyun Wang, and Anette M. Karlsson ${ }^{a)}$ \\ Department of Mechanical Engineering, University of Delaware, Newark, Delaware 19716
}

\begin{abstract}
The microstructures of exoskeletons from Homarus americanus (American lobster) and Callinectes sapidus (Atlantic blue crab) were investigated to elucidate the mechanical behavior of such biological composites. Image analyses of the cross-sectioned exoskeletons showed that the two species each have three well-defined regions across the cuticle thickness where the two innermost regions (exocuticle and endocuticle) are load bearing. These regions consist of mineralized chitin fibers aligned in layers, where a gradual rotation of the fiber orientation of the layers results in repeating stacks. The exocuticle and endocuticle of the two species have similar morphology, but different thicknesses, number of layers, and number of stacks. Mechanics-based analyses showed that the morphology of the layered structure corresponds to a nearly isotropic structure. The cuticles are inter-stitched with pore canal fibers, running transversely to the layered structure. Mechanics-based analyses suggested that the pore canal fibers increase the interlaminar strength of the exoskeleton.
\end{abstract}

\section{INTRODUCTION}

Exoskeletons are the hard, external covering of arthropods such as crustaceans (e.g., lobsters, crabs, and shrimp) and insects (e.g., beetles). Many exoskeletons have outstanding material properties ${ }^{1}$ with multiple functions such as carrying the body weight, serving as a filter for chemicals and environmental exposure, and providing armor against enemies. ${ }^{2}$ To achieve this, nature primarily uses one material - the biopolymer chitin - to develop a complex hierarchical structure, together with connective tissue consisting of proteins and minerals. Variations in the morphology of the chitin network and mineralization within the structure lead to a range of properties. $^{1}$

Arthropod exoskeletons are commonly composed of three major regions including epicuticle, exocuticle, and endocuticle (Fig. 1). The outmost region, the epicuticle, is thin $(2-4 \mu \mathrm{m})$ compared to the other regions. It is a waxy layer lacking chitin, acting as a diffusion barrier. The exocuticle and endocuticle are mainly made of the chitin-protein marcofibrils, with minerals deposited in between for species with calcified exoskeletons. ${ }^{3.4}$ Chitin is a natural polysaccharide that is commonly found in most arthropods. These chitin polymers with their asso- ciated proteins form microfibrils. ${ }^{3,5}$ Bundles of microfibrils form the so-called "macrofibrils," which can be observed under electron microscopy. The deposited minerals in calcified samples of crustaceans include calcite, vaterite, and hydroxyapatite. ${ }^{3}$ The exocuticle and endocuticle are the load-carrying structure in the cuticle ${ }^{3}$ and vary in thickness, depending on species.

Although the shell of nacre has received significant attention in the last decade (e.g., Refs. 6-9), the architecture and mechanical behavior of most arthropods exoskeletons are not well-understood. By investigating and developing an understanding for the multilayered "biomineral" composite structure of arthropods, guidelines and ideas for man-made multifunctional structures with superior properties may be reached. ${ }^{10,11}$ In this work, we investigated the exoskeletons of two crustaceans, the Callinected sapidus (Atlantic blue crab) and Homarus americanus (American lobster). We first examined the microstructure of these biologically formed composites and adopted simple mechanics based models to elucidate the mechanical response. The microstructure was obtained through investigation of representative samples using electron microscopy and image analysis, reviewing and extending the current understanding of the microstructure. The mechanical response was evaluated by adapting and extending suitable models developed for composite materials. The results revealed important and novel information about the mechanical response of exoskeletons from arthropods. 


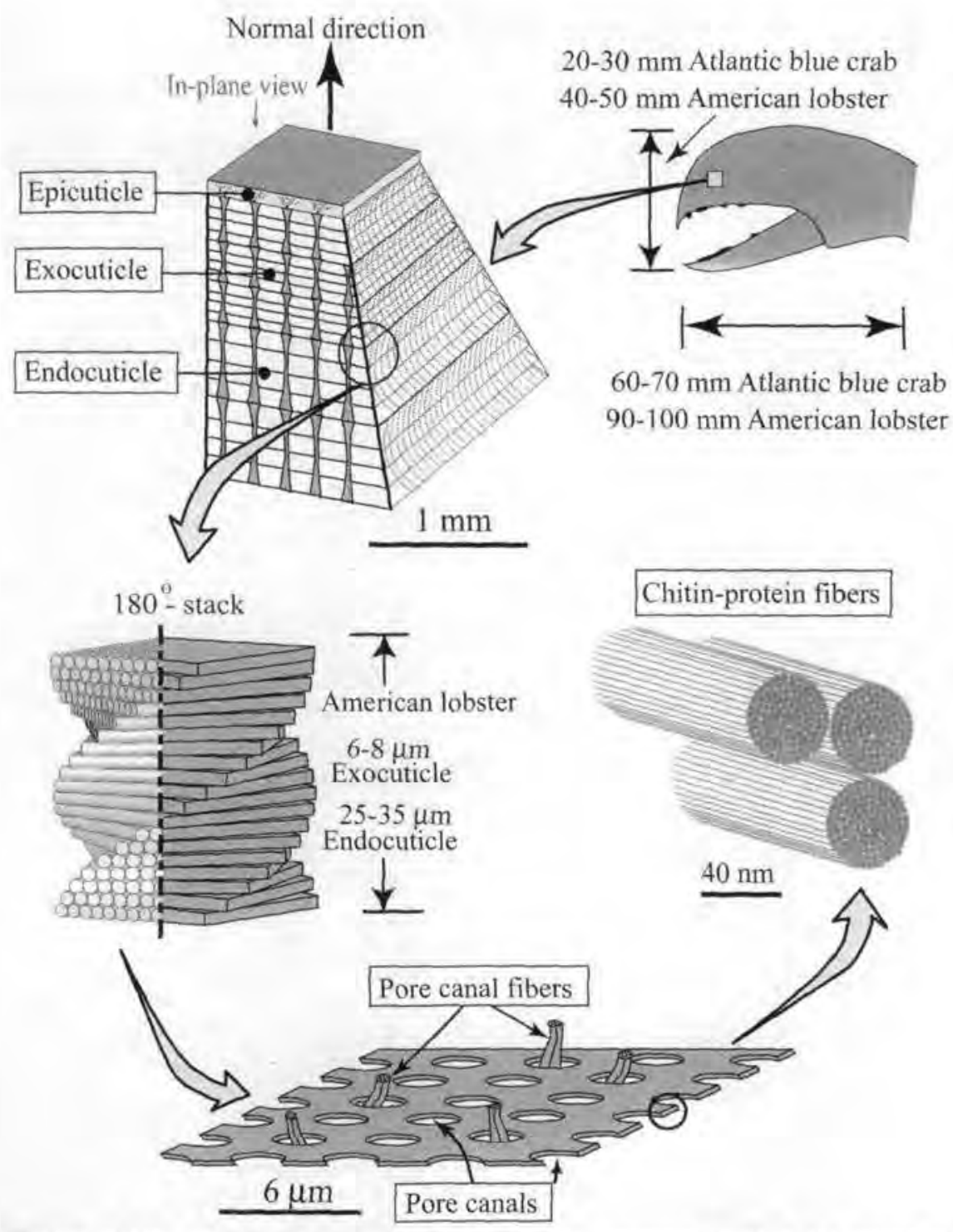

FIG. I. Schematic illustrations of the hierarchical structure from millimeter to nanometer levels of the cuticle of a Homarus americanus (American lobster) and Callinectes sapidus (Atlantic blue crab). The outer layer (epicuticle) acts as a diffusion barrier. The exocuticle and endocuticle layers are load bearing structures built of mineralized fibrous chitin-protein with similar morphology. The structure in the endocuticle has larger dimensions than that in the exocuticle. Drawings are in different scales, indicated by the associated scale bars.

\section{IMAGE ANALYSIS}

\section{A. Sample preparation}

Representative samples $(30 \times 20 \mathrm{~mm}$ cuticle strips $)$ were taken from claws and dorsal carapace of Callinected sapidus and Homarus americanus and prepared for image analysis. The samples were divided into three groups with 10 samples per group for each individual species, and underwent either air drying, ethanol dehydration, or decalcification.

Samples from the first group were air-dried in a regular desiccator for two to three days.

Samples from the second group were fixed in $70 \%$ ethanol alcohol (ETOH) overnight, then dehydrated in ascending grades of ETOH (95\% and $100 \%$ ) and finally in $100 \%$ ETOH with a molecular sieve (Grade 564 Type 3A, Fisher Scientific, Pittsburgh, PA) to absorb all the moisture from the ETOH, for 30 min each. In the last step, critical point dehydration was conducted, using a dryer (Autosamdri 815B, Seris A, Tousimis Research Corporation, Rockville, MD) to prevent the samples from being damaged due to large tension force in the liquid-gas interface during traditional drying. [Critical point dehydration (CPD) is a method of drying tissue without damaging the structure of the delicate specimen. Typically large tension forces develop in the liquid-gas interface during drying. At the critical point (associated with critical temperature and pressure), the liquid and the gas phase have the same density; thus a distinct interface will not be generated, preventing tension forces from developing.] 
Samples from the third group were first fixed overnight in $2.5 \%$ glutaraldehyde in $0.2 \mathrm{M}$ sodium cacodylate buffer $(\mathrm{pH} 7.4)$ and then decalcified in 10\% EDTA (edetic acid) in $0.1 \mathrm{M}$ Tris- $\mathrm{HCl}(\mathrm{pH} \mathrm{7.0)}$ for $2-3$ weeks until the cuticle was soft. The decalcified samples were rinsed in $0.2 \mathrm{M}$ sodium cacodylae buffer three times for $10 \mathrm{~min}$ each and then fixed in $0.5 \% \mathrm{OsO}_{4}$ for $2 \mathrm{~h}$. After being rinsed in de-ionized water three times for $10 \mathrm{~min}$ each, samples were dehydrated in ascending ETOH series through $25 \%, 50 \%, 70 \%$, and $95 \%$ for 15 min each. Dehydration was further carried out with $100 \% \mathrm{ETOH}$ for $30 \mathrm{~min}$ followed by additional $30 \mathrm{~min}$ in $100 \% \mathrm{ETOH}$ with a molecular sieve. Finally, critical point dehydration was performed as described for the previous group.

Fracture surfaces were made from the prepared samples using forceps and a single-edged razor blade to expose either the cross sections or planes parallel to the surface of the exo- or endocuticle. Because of the difficulty in controlling the fracture plane, oblique fracture sections were commonly obtained. These fractured samples were sputtercoated with gold/palladium for 2.5-3 min and then observed with scanning electronic microscopy (SEM) using two different microscopes (JSM-7400, JEOL, Tokyo, Japan and Hitachi S-4700, Tokyo, Japan).

\section{B. Microstructural observations}

The exoskeletons of the investigated arthropods are found to be layered structures, divided into three distinct regions: epicuticle, exocuticle, and endocuticle (Fig. I). Transverse pore canals and pore canal fibers are also dominant features, running perpendicular to these (inplane) regions. The key features and measurements of the structures from both species are summarized in Table I and will be discussed in the following.

\section{Layered structure}

The exoskeletons consist of three distinct regions (Fig. 1). The outermost region, the epicuticle, is much thinner than the other regions, with a thickness of approximately 2-4 $\mu \mathrm{m}$. Cross-sectional images of the epicuticle from the air-dried samples (without decalcification) reveal a dense structure, where the microscopic features are hard to distinguish (not shown for brevity). However, in decalcified samples of both species [Figs. 2(b) and 3(b)], closely packed fibrous structures perpendicular to the external surface are observed. Since this layer is not loading-bearing, it will not be studied further in this work. ${ }^{3}$

Beneath the epicuticle are the two major load-bearing regions: the exocuticle and endocuticle (Fig. 1). These two regions have similar morphology but have different thicknesses. The exocuticle is approximately 150$180 \mu \mathrm{m}$ thick for the Homarus americanus claw [Fig. 2(c)] and 40-50 $\mu \mathrm{m}$ for the Callinected sapidus claw [Fig. 3(c)]. The thickness of the endocuticle is approximately 3-4 times that of the exocuticle for Homarus americanus and 6-8 times that for Callinected sapidus [Figs, 2(a) and 3(a)]. Similar to the epicuticle, a detailed fiber structure cannot be detected in the air-dried samples but can be clearly observed in the decalcified samples. Measurements from the SEM images of the decalcified samples indicate that the diameter of the fibers is approximately $30-40 \mathrm{~nm}$. These fibers are the chitinprotein fibrils, termed macrofibrils. ${ }^{3,11}$

The exocuticle and endocuticle consist of multiple chitin-protein layers, with the chitin-protein macrofibrils arranged parallel to each other in each layer. The chitinprotein layers stack successively on each other, with each layer rotated with a small angle of rotation about the normal direction relative to its adjacent layer, schematized in Fig. 4(c). This stacking sequence results in an apparent parabolic pattern in oblique sections of the $\mathrm{cu}$ ticles (Fig. 4). The parabolic appearance is a purely geometric illusion and has caused some misinterpretation in the literature. What appears as a parabolic "curve" is in fact just a sequence of oblique cuts of macrofibrils in a series of layers, where one "full parabola" corresponds to

TABLE 1. Structure comparison between Homarus americanus and Callinected sapidus claw, based on image analysis.

\begin{tabular}{|c|c|c|c|}
\hline Region & Property & Homarus americanus & Callinected sapidus \\
\hline \multirow[t]{2}{*}{ Epicuticle } & Thickness & $2-4 \mu \mathrm{m}$ & $2-4 \mu \mathrm{m}$ \\
\hline & Pattern & Fibers aligned perpendicular to the surface & $\begin{array}{l}\text { Fibers aligned perpendicular } \\
\text { to the surface }\end{array}$ \\
\hline \multirow[t]{4}{*}{ Exocuticle } & Thickness & $150-180 \mu \mathrm{m}$ & $40-50 \mu \mathrm{m}$ \\
\hline & Pattern & Bouligand architecture & Bouligand architecture \\
\hline & $180^{\circ}$-stack & $6-8 \mu \mathrm{m}$ & $-3 \mu \mathrm{m}$ \\
\hline & Fiber volume fraction & High & High \\
\hline \multirow[t]{4}{*}{ Endocuticle } & Thickness & $600-700 \mu \mathrm{m}$ & $300-400 \mu \mathrm{m}$ \\
\hline & Pattern & Bouligand structure & Bouligand structure \\
\hline & $180^{\circ}$-stack & $25-35 \mu \mathrm{m}$ & $11-12 \mu \mathrm{m}$ \\
\hline & Fiber volume fraction & Low & Low \\
\hline \multirow[t]{3}{*}{$\begin{array}{l}\text { Pore canal and pore } \\
\text { canal fiber }\end{array}$} & Pattern & $\begin{array}{l}\text { Transverse through the thickness of } \\
\text { the exo- and endocuticles }\end{array}$ & $\begin{array}{c}\text { Transverse through the thickness } \\
\text { of the exo- and endocuticles }\end{array}$ \\
\hline & Density & $0.13-0.16 \mu \mathrm{m}^{-2}$ & $0.22-0.50 \mu \mathrm{m}^{-2}$ \\
\hline & Fiber diameter & $0.1-0.5 \mu \mathrm{m}$ & $0.5-2 \mu \mathrm{m}$ \\
\hline
\end{tabular}




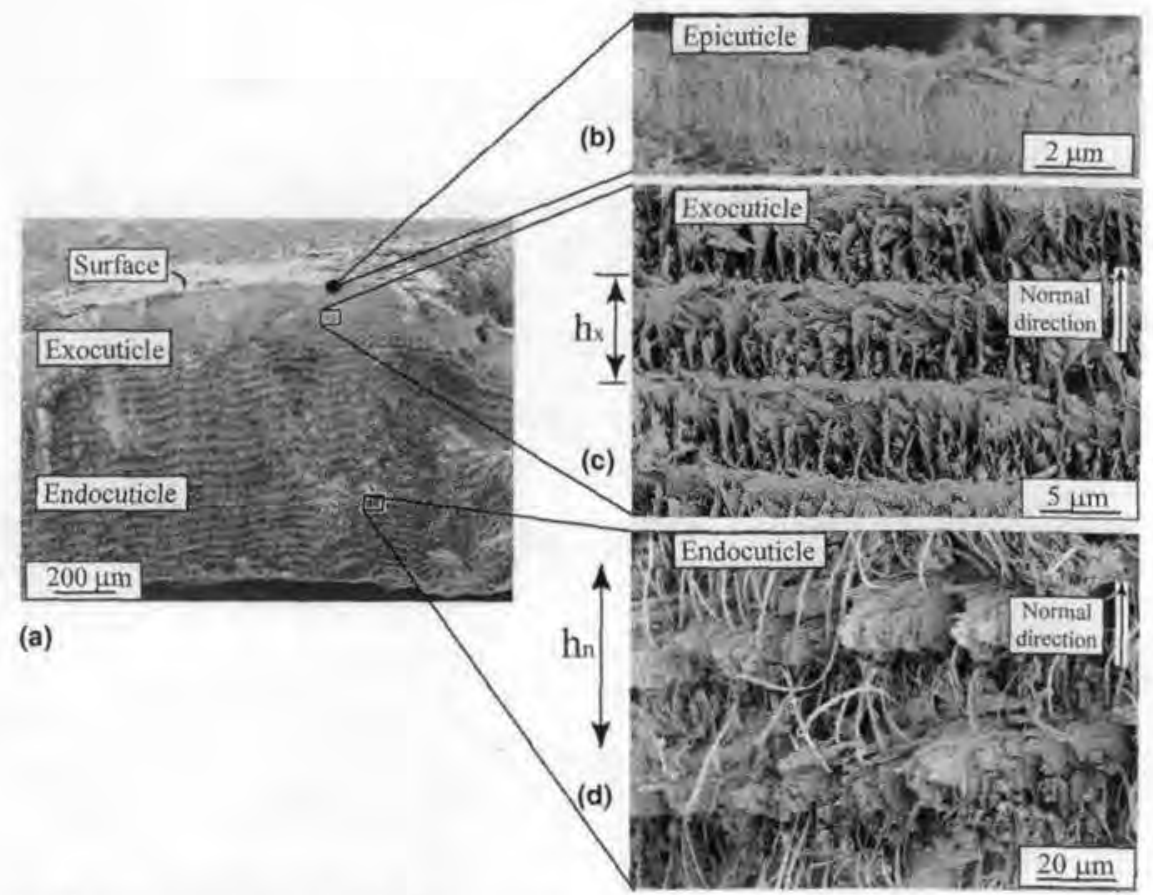

FIG. 2. SEM images of a Homarus americanus cuticle: (a) overview of the cross-section, (b) epicuticle (2-4 $\mu \mathrm{m}$ thick), (c) exocuticle (150$180 \mu \mathrm{m})$, and (d) endocuticle $(600-700 \mu \mathrm{m}) \cdot h_{x}(6-8 \mu \mathrm{m})$ and $h_{\mathrm{n}}(25-35 \mu \mathrm{m})$ represent the thickness of the $180^{\circ}$-stack in exocuticle and endocuticle, respectively.

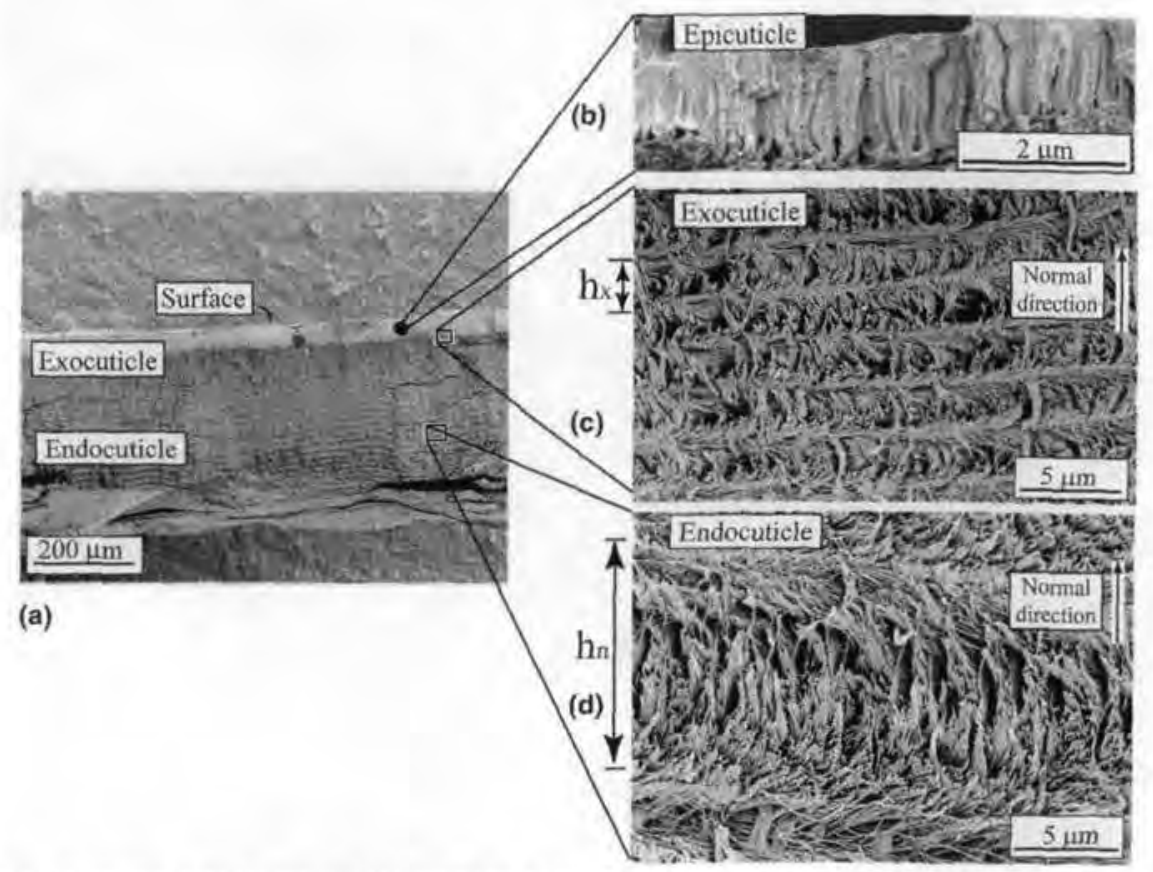

FG. 3. SEM images of a Callinectes sapidus cuticle: (a) overview of the cross-section, (b) epicuticle $(2-4 \mu \mathrm{m}$ thick), (c) exocuticle (30-50 $\mu \mathrm{m})$, and (d) endocuticle $(250-300 \mu \mathrm{m}) . h_{x}(\sim 3 \mu \mathrm{m})$ and $h_{\mathrm{n}}(11-12 \mu \mathrm{m})$ represent the thickness of the $180^{\circ}$-stack in exocuticle and endocuticle, respectively.

$180^{\circ}$ rotation of the layers, as was visualized in Fig. 4 (referred to as a " $180^{\circ}$ stack"). This structural pattern is termed a "helicoidal structure," which was first suggested by Bouligand ${ }^{12}$ and has been explored by GiraudGuille. ${ }^{13}$

\section{Pore canals and pore canal fibers}

In addition to the layered helicoidal structure, there are distinct through-the-thickness structural features: the pore canals (PC) and associated pore canal fibers (PCF). 


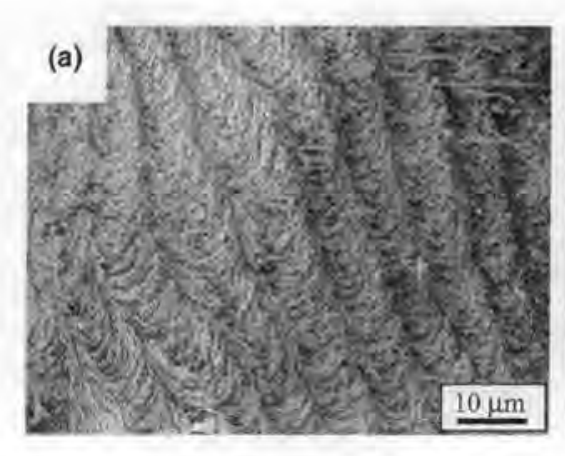

(c)
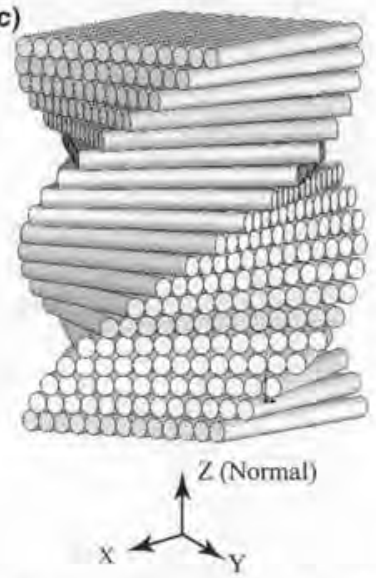
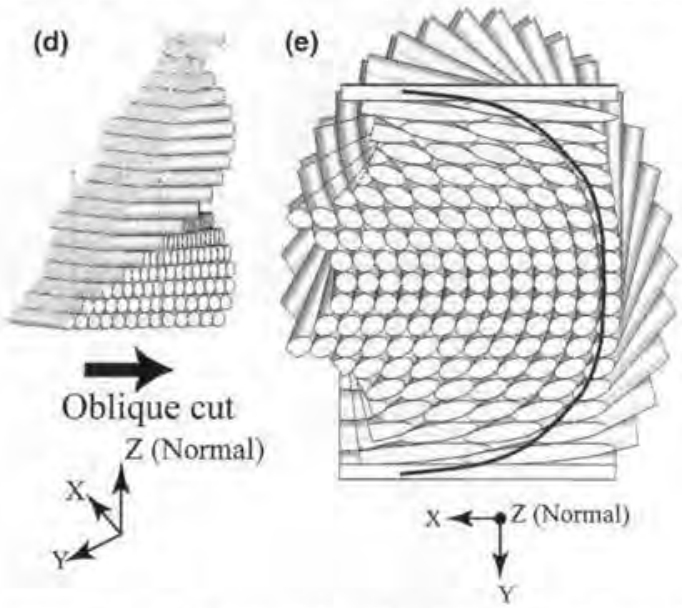

FIG. 4. Helicoidal pattern (Bouligand-structure) of the chitin-protein fibers in exocuticle and endocuticle: (a) A parabolic pattern can be seen in a SEM image of an oblique section of the exocuticle from the ethanol dehydrated Homarus americanus claw. (b) Fiber orientations are shown in a SEM image of a cross-section of the decalcified endocuticle from a Homarus americanus claw. Arrows indicate the successive rotation of chitin-protein fibers in neighboring layers. Transverse fibers (PCF = pore canal fibers) are clearly seen. (c) In a schematic representation the Bouligand pattern, each layer represent parallel fibers, and the layers are successively rotated unidirectionally relative to their adjacent layers. (d) An oblique cut is made in the schematic Bouligand block. (e) The side view of the oblique cut displays the parabolic pattern seen in (a) and (b).

Fibers termed as pore canal fibers are present in both species, orientated through (across) the thickness of the exoskeletons (Fig. 5). Similar to the in-plane layers, the pore canal fibers consist of closely packed macrofibrils with a diameter of about $30 \mathrm{~nm}$. These macrofibrils are thought to be constructed by chitin-protein, ${ }^{14,15}$ although their exact chemical compositions are not determined in this study. The pore canal fibers are located in the spaces enclosed by the pore canals, which also extend through the thickness of the exoskeletons. The pore canals are formed by aligned oval-shaped holes in the chitin-protein layers. They are evenly distributed in both exocuticle and endocuticle [Figs, 6(a) and 6(b)] and run perpendicular to the layers. The oval shaped holes are formed by opening or closing the branches of chitin-protein macrofibrils in each layer, which is clearly demonstrated in decalcified samples [Fig. 6(b)]. Pore canals and pore canal fibers are found in both species investigated (Fig. 6). Measurement of in-plane SEM images indicates that the Callinected sapidus has a higher pore canal density $\left(0.22-0.25 \mu \mathrm{m}^{-2}\right)$ than that of the Homarus americanus $\left(0.13-0.16 \mu \mathrm{m}^{-2}\right)$. However, the size of the pore canal fibers in Homarus americanus is larger than that in Callinected sapidus
[Fig. 6(b) versus Fig. 6(c)]. [Samples from the two species were processed differently in the case shown in Fig. 6: Homarus americanus (decalcification) and Callinected sapidus (air drying). Although decalcification increased the size of the pores by removing the minerals, the number and the density of the pore canals were not affected.]

In the exocuticle, the pore canal fibers are well embedded in and in close contact with the chitin-protein matrix through which they run, as can be seen from the undecalcified samples of both species [Fig. 7(a)]. However, in the endocuticle, the pore canal fibers may not necessarily be well-embedded in their surrounding matrix for either species [Fig. 7(b)].

The pore canals and the pore canal fibers are believed to have at least two functions: transport of impregnating materials during molting process (e.g., Refs. 3, 14, and 16) and enhancement of the mechanical strength of the cuticle, as noted by Mutvei. ${ }^{17}$ However, the latter has, to our knowledge, not been investigated. We will discuss the effect of pore canal fibers on the mechanical behaviors of the exoskeleton in the next section.

Due to the helicoidal arrangement of layered structure, 

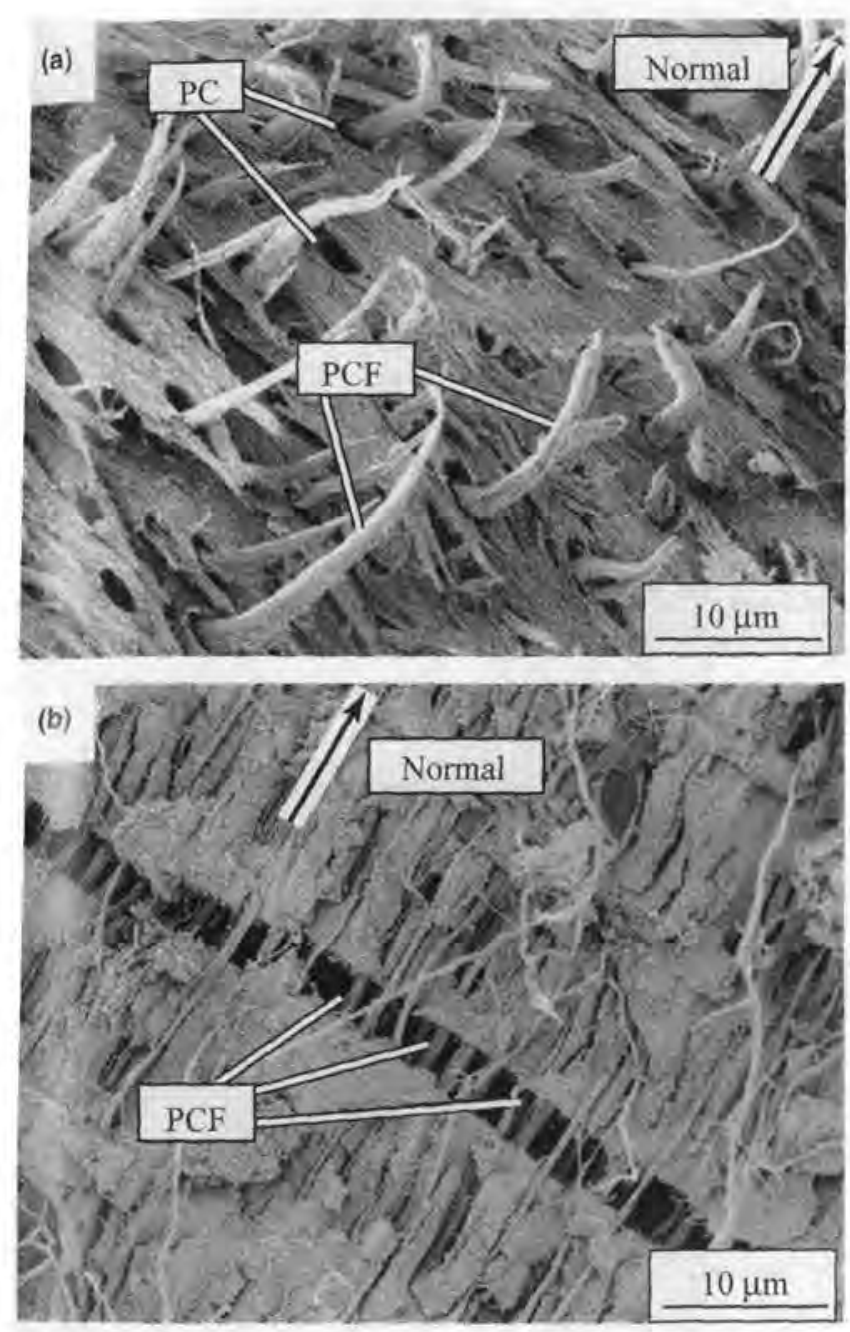

FIG. 5. SEM images of pore canals (PC) and PCF. Arrow marks the normal direction of the cuticle external surface in each image. (a) In-plane view of a representative sample from a Homarus americanus claw. (b) Cross-sectional view of a sample from a Callinectes sapidus carapace,

the shape of the pore canals can be described as a twisted ribbon structure. ${ }^{3,14,16}$ Furthermore, in the oblique sections, a parabolic pattern of the pore canals can be observed, which again is a geometric illusion caused by the helicoidal structure (Fig. 8). We note that it has been suggested that the pore canals and the pore canal fibers are "cross-linked," forming a similar pattern as the chitin-protein macrofibril layers (e.g., Ref. 18). We believe that this may be a misinterpretation due to the "twisted ribbon" appearance of the pore canals. Our analyses based on SEM images [Figs. 4(b), 5, and 7] indicate that the pore canal fibers are indeed straight and are not cross-linked to each other, as illustrated in Fig. 8.

\section{MECHANICS-BASED ANALYSIS}

Based on the experimental observations, it is evident that the exoskeletons can be considered a biomineralized composite, characterized by a natural fiber-reinforced, laminar composite. The exoskeletons consist of proteins and minerals that serve as a continuous matrix, reinforced with chitin fibers of high tensile stiffness and strength. Successive chitin-protein layers (lamina) are stacked together in a helicoidal structure to form either the exo- or endocuticle.

We will now focus on the mechanical response from the structural features observed in the exoskeletons. First, we discuss the mechanical response associated with the layered structure, followed by a discussion about the mechanical enhancement resulting from the pore canal fibers. The purpose of the analyses that follow is to develop a qualitative understanding of the mechanical response, within a linear-elastic assumption. The developed models are mainly based on the morphological results obtained from our imaging analysis, and the material properties used in the models are estimated from literature data due to lack of experimental measurements of the exoskeletons. More experimental data on damage mechanisms, interfacial molecular interaction among various compositional components ${ }^{19.20}$ are needed to develop a more detailed multiscale model where nonlinear effects can be incorporated. ${ }^{21-23}$ This is beyond the scope of the current study and left for future investigations.

\section{A. Layered structure}

\section{Classic laminate theory}

A model of the representative repeating unit (unit cell) of the layered structure, which consists of $180^{\circ}$ accumulated rotation of layers, a " $180^{\circ}$-stack," is developed for the mechanics-based analysis. In one layer (lamina), the principal material direction is along the fiber direction. Due to the high stiffness of the chitin fibers, the elastic modulus is assumed higher in the principal material direction than in any other directions. To form the helicoidal architecture observed experimentally, laminae are stacked together with the principal material direction of each lamina rotating a small angle about the normal direction relative to its neighboring lamina [Fig. 9(a)]. For simplicity, we do not explicitly include the local contribution of the holes forming the pore canals, but instead incorporate them into the overall stiffness for each lamina. Furthermore, using experimental observations, we assume a constant thickness of each lamina and a constant angle of relative rotation in the laminate for the exocuticle and endocuticle, respectively, although the thickness and angle of rotation vary between exo- and endocuticles, and between species. With these simplifications, the exoskeletons can be characterized as laminated (layered) composites, using theories from the literature, e.g., Ref. 24.

The global coordinate system $(x, y, z)$ is shown in Fig. 9(b). The principal directions of the lamina (local 

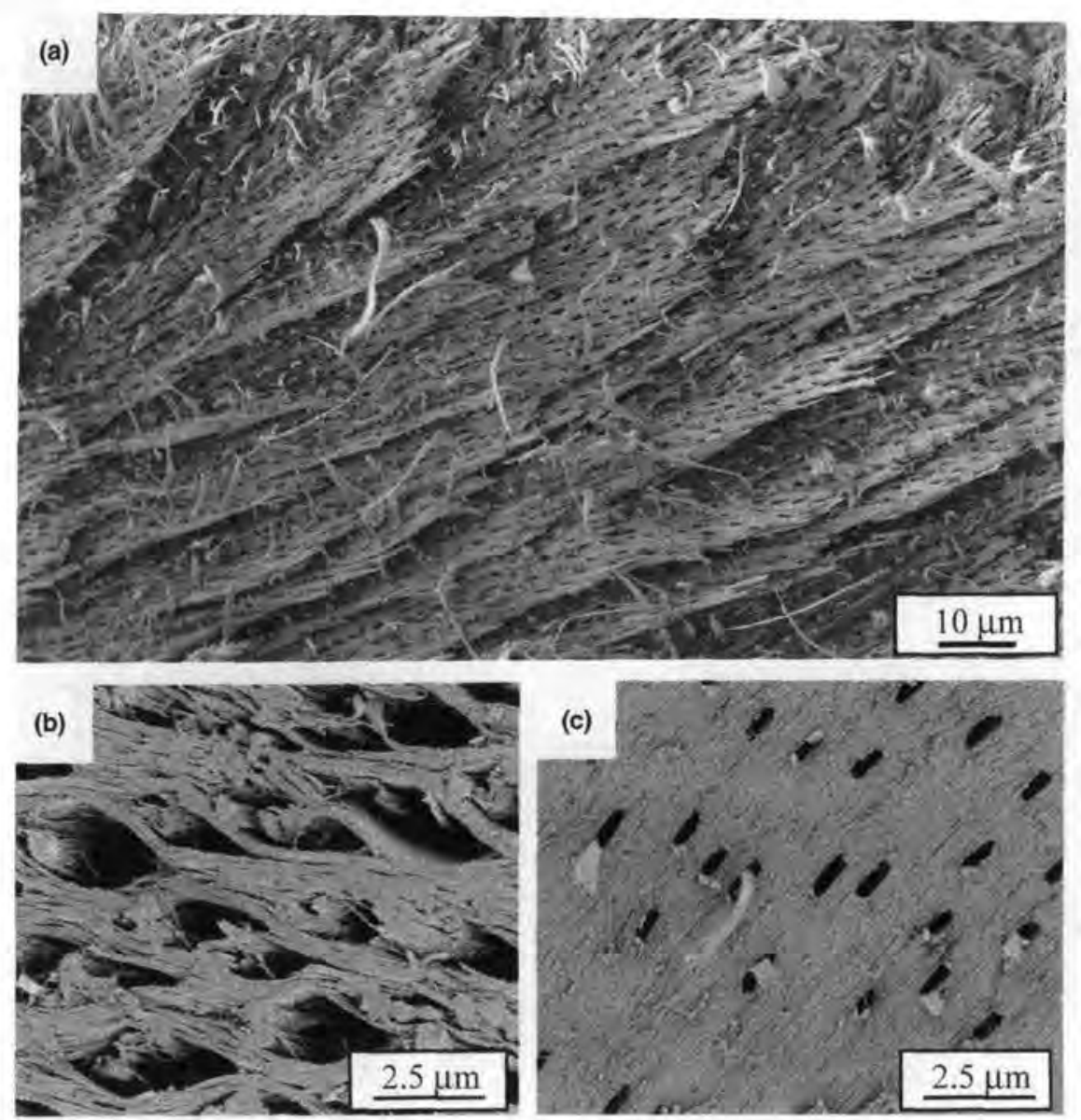

FIG. 6. In-plane views of the endocuticles exposing the PC and PCF. (a) Part of the cuticle is peeled off to expose this region showing that successive planes stack together to form the endocuticle (Homarus americanus). (b) PC and associated PCF are shown in a decalcified endocuticle from the Homarus americanus. The PC appear larger in the decalcified samples due to removal of minerals. (c) PC and associated PCF are shown for air-dried endocuticles Callinected sapidus.

coordinate system) are denoted 1 and 2 , where 1 corresponds to the fiber direction of an orthotropic material [Fig. 9(a)]. The out-of-plane direction of the lamina is parallel to the $z$ axis and is denoted 3. For the lamina, plane stress conditions is assumed. With this approximation, the remaining non-vanishing stresses are $\sigma_{1}, \sigma_{2}$, and $\tau_{12}$, where $\sigma$ and $\tau$ denote the normal stress and shear stress, respectively. The corresponding engineering constants are the elastic moduli $E_{1}$ and $E_{2}$ in the 1 and 2 directions, respectively; $\nu_{12}$ is Poisson's ratio and $G_{12}$ is the shear modulus in the plane.

Due to the $180^{\circ}$ successive rotation along with the assumptions of constant lamina thickness and constant relative rotation, each layer in the $180^{\circ}$-stack model will have a counterpart on the other side of the middle plane; i.e., a lamina at $z=z_{1}$ rotated $\theta_{1}$ about the normal axis will have its counterpart at $z=-z_{1}$ with $-\theta_{1}$ rotation (negative rotation). Thus, the $180^{\circ}$-stack model and also the exoskeleton can be categorized as a regular antisymmetric laminate. Here, the resultant in-plane normal forces $N_{x}$ and $N_{y}$ and in-plane shear force $N_{x y}$, as well as the bending moments on the surfaces with normals in the $x$ and $y$ directions $M_{x}$ and $M_{y}$ and the twist of the laminate $M_{x y}$, can be related to the normal strains $\epsilon_{s}^{0}, \epsilon_{y}^{0}$ and shear strain $\gamma_{x y}^{0}$, all at the mid-plane, and the curvatures $K_{x}, K_{y}$ and $K_{x y}$ according to ${ }^{24,25}$ (Fig, 10)

$$
\mathbf{N}=\mathbf{A} \epsilon+\mathbf{B} \boldsymbol{N}, \quad \mathbf{M}=\mathbf{B} \epsilon+\mathrm{D}_{\boldsymbol{k}},
$$

where $\mathbf{N}, \mathbf{M}, \boldsymbol{\epsilon}$ and $\boldsymbol{\kappa}$ are the vectors for normal force, bending moment, strain, and curvature as defined in the Appendix. Furthermore, $\mathbf{A}$ is the extensional stiffness matrix, $\mathbf{B}$ is the bending-extension coupling stiffness matrix, and $\mathbf{D}$ is the flexural stiffness matrix, defined in the Appendix.

The constitutive relationship presented in Eq. (1) results in a coupling between stretching and twisting, and between bending and shearing, manifested by the coupling terms in the $\mathbf{B}$ matrix $B_{16}$ and $B_{26}$, defined in the Appendix.

An idealized structure, consisting of a laminate with an infinite number of laminae and infinitesimal rotation angle between each lamina, will give an upper bound for 

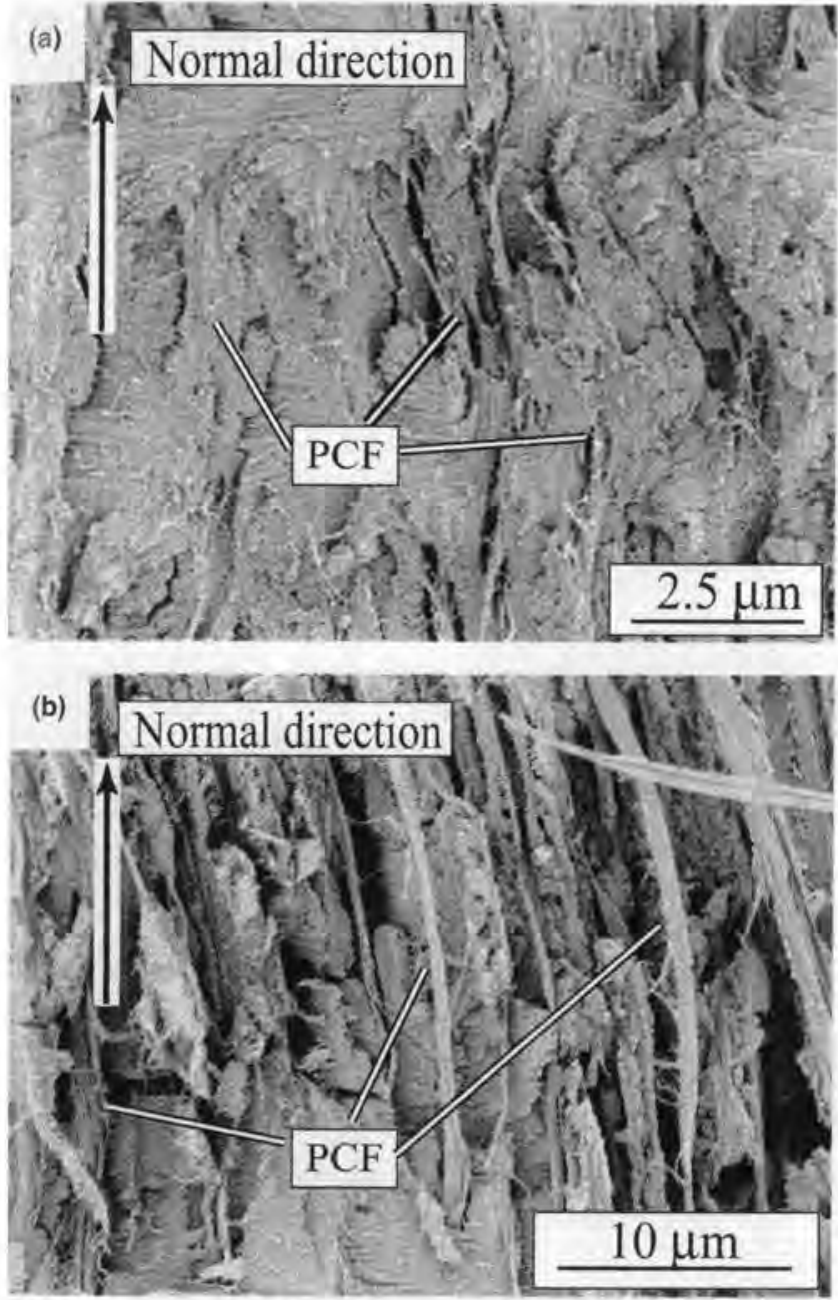

FIG. 7. Interactions between PCF and surrounding matrix shown in SEM images of the undecalcified (ethanol dehydrated) samples of Homarus americanus: (a) in the exocuticle region, the transverse PCF are well-embedded and in close contact with their surrounding matrix, and (b) in the endocuticle region, the transverse PCF are not necessarily well-embedded in their surrounding matrix.

how an $180^{\circ}$-stack responds. To achieve this, the thickness of the lamina can be assumed infinitesimally small, $d h$ and the components in the stiffness matrixes can be rewritten in integrated forms as

$$
\begin{aligned}
\mathrm{A}_{i j} & =\int_{-H}^{H}\left(\bar{Q}_{i j}\right)_{\mathrm{k}} d h, B_{i j}=\int_{-H}^{H}\left(\bar{Q}_{i j}\right)_{\mathrm{k}} h d h, \\
D_{i j} & =\int_{-H}^{H}\left(\bar{Q}_{i j}\right)_{\mathrm{k}} h^{2} d h,
\end{aligned}
$$

where $2 H$ is the total thickness of the laminate and $\bar{Q}_{i j}$ are the transformed stiffnesses defined in the Appendix. The resulting stiffness matrices are similar to that of a regular antisymmetric laminate, except that $A_{11}=A_{22}$, corresponding to the in-plane isotropy. Thus, for a structure approaching infinitely many laminae and infinitely small rotation angle, the laminate is a quasi-isotropic laminate. However, coupling between in-plane loading and bending still exists.

\section{Structural response}

To elucidate the response of the exoskeleton, we now investigate the constitutive behavior for a structure with thickness $(2 H=30 \mu \mathrm{m})$, approximately corresponding to the thickness of a " $180^{\circ}$-stack" observed for the lobster samples, for a range of numbers of lamina $(N)$, thus with different relative rotation angles.

The material properties are determined according to the following. The matrix and fibers are each assumed isotropic. The stiffness for the crystalline chitin is reported to vary in the range of $10-100 \mathrm{GPa}^{26}$ Mechanical properties are not readily available for the calcified protein matrix, primarily due to challenges associated with measuring them and the sensitivity to factors such as water content and extent of calcification. However, the elastic modulus of the cuticle matrix of Schistocerca varies from $0.1 \mathrm{GPa}$ (Apodeme) to $2 \mathrm{GPa}$ (Tibia) in different locations. ${ }^{27-30}$ Poisson's ratio for both materials is assumed to be 0.25 . The shear modulus can be determined from $G=E / 2(1+v)$. The chitin volume fraction is approximately $15-30 \%$ in the cuticle. ${ }^{29,30}$ Here, we select $20 \mathrm{GPa}$ as elastic modulus for chitin, and $1 \mathrm{GPa}$ for the protein matrix. Corresponding shear moduli will be $8 \mathrm{GPa}$ for chitin and $0.4 \mathrm{GPa}$ for protein. Moreover, we assume a $30 \%$ chitin volume fraction and estimate the properties of the bio-composite by using Hahn's equations; see the Appendix for details. ${ }^{24}$ The resulting parameters are shown in Table II. (The purpose of the following calculations is to investigate qualitatively how the structure behaves. In a future study, we plan to conduct testing to establish the true mechanical properties.)

Table III shows how the normalized laminate properties, based on Eq. (A3), evolve with the number of laminae, within a $180^{\circ}$-stack laminate. With increasing number of laminae in the laminate, normalized $\hat{A}_{11}$ and $\hat{A}_{22}$ converge to one value; that is, the extensional stiffness approaches an isotropic response. Isotropy is obtained with infinitely many laminae, corresponding to the analytical solution above [Eq. (2)]. Isotropy is generally preferred since it provides equal structural behavior in all directions.

Based on image analyses of the exoskeleton samples from the Homarus americanus and Callinected sapidus, each $180^{\circ}$-stack consists of approximately $100-200$ laminae in the endocuticle, assuming that one lamina is composed of parallel macrofibrils in one plane. From the results in Table III, this corresponds to a high level of isotropy:

$$
\hat{A}_{11} \approx \hat{A}_{22} \quad ; \quad \hat{A}_{66} \approx \frac{\hat{A}_{11}-\hat{A}_{12}}{2} .
$$




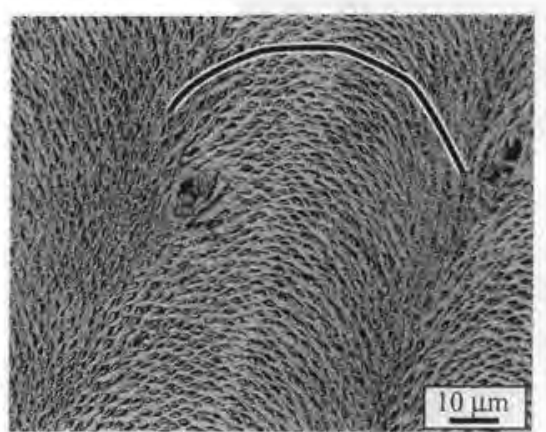

(a) (b)

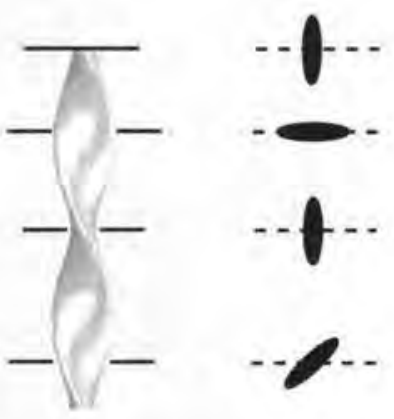

(c)

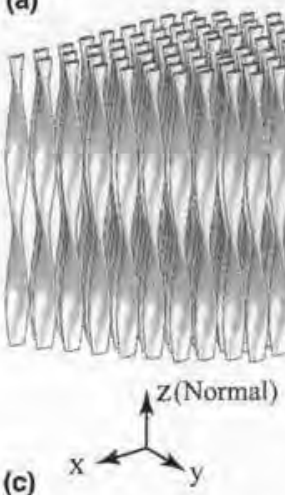

FIG. 8. Helicoidal pattern (Bouligand-structure) of the PC and its corresponding illustrations: (a) SEM image of an oblique section of the endocuticle from the decalcified Homarus americanus claw, highlighting the parabolic pattern of the PC (shown as densely distributed black holes in the image); (b) "twisted ribbon effect" of a pore canal as a result of the successive rotation of the horizontal layers (left side) and the cross sections of the pore canal at different positions (right side); (c) parallel PC running toward the surface along the normal direction; (d) oblique cut of the PC; and (e) in-plane view of the oblique cut displays the parabolic pattern of the PC seen in (a).
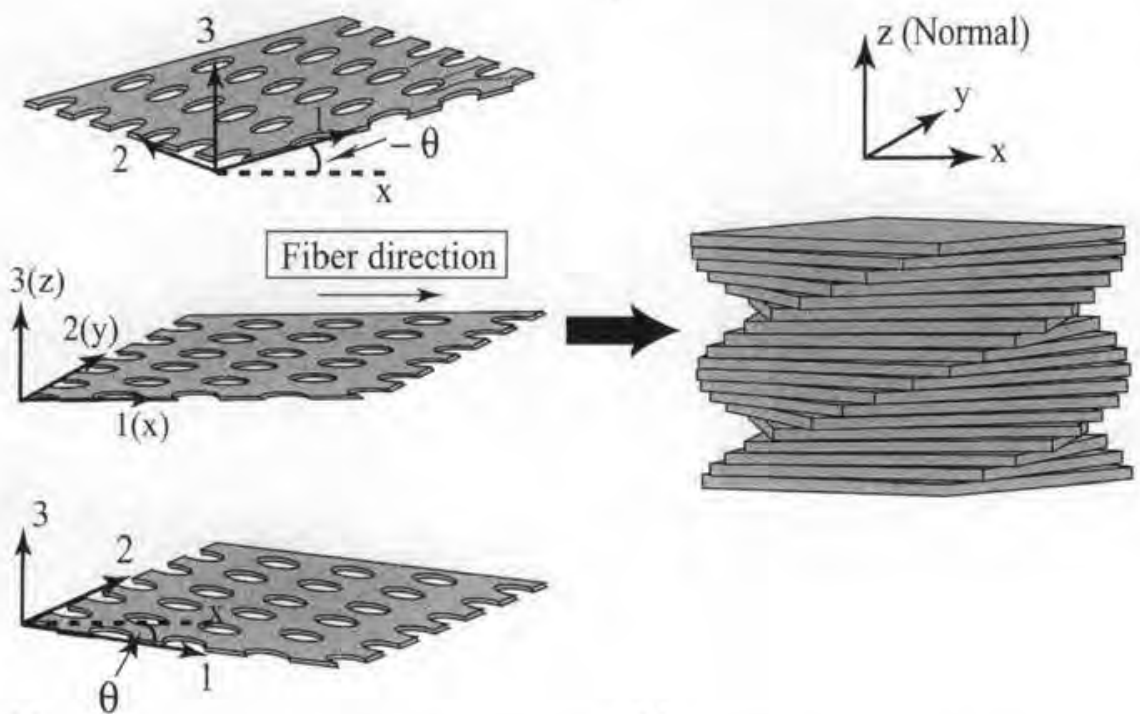

(a)

(b)

FIG. 9. Schematic illustrations of the $180^{\circ}$-stack composed of successively rotated laminae with the global coordinate system $[x, y, z]$. The local coordinate system $[1,2,3]$ is attached to individual lamina in the laminate and rotates about the $z$ axis (e.g., $\theta$ or $-\theta$ ).

A similar symmetry is not found in the flexural stiffness matrix, due to the nature of the antisymmetric laminate in our model.

As discussed previously, the exo- and endocuticles share the same structural characteristics, with similar overall mechanical characteristics, as analyzed above. However, there are distinct differences between the two regions with respect to structural parameters, resulting in different mechanical responses. The $180^{\circ}$-stack model we developed is the basic repetitive unit of the structures, 


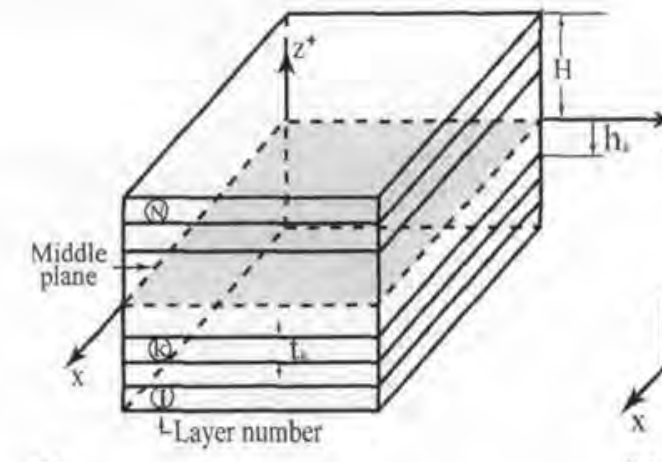

(a)

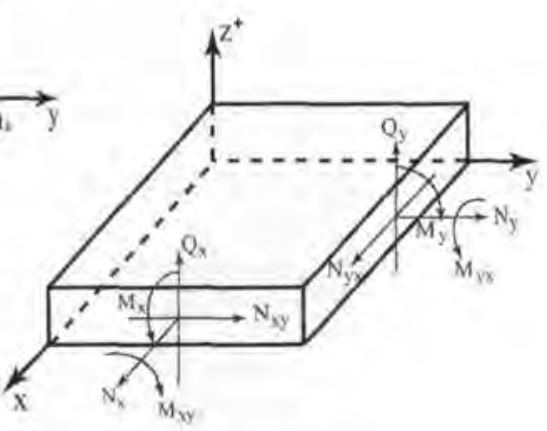

(b)

FIG. 10. (a) Parameters of the laminate model and (b) forces and moments in a single layer. The total thickness of the laminate is $2 \mathrm{H}$ and the thickness of the $k$ th layer is $t_{k}$. For a single layer in (b), all stress resultants and stress couples are shown in their positive directions (on two sides only for simplicity).

TABLE II. Parameters and mechanical properties of one layer of fiber-matrix.

\begin{tabular}{ccccc}
\hline \hline$E_{1}$ & $E_{2}$ & $v_{12}$ & $G_{12}$ & $2 H$ \\
\hline $6.7 \mathrm{GPa}$ & $1.56 \mathrm{GPa}$ & 0.25 & $0.71 \mathrm{GPa}$ & $30 \mu \mathrm{m}$ \\
\hline \hline
\end{tabular}

TABLE III. Numerical results of normalized stiffnesses of exoskeleton $180^{\circ}$-stack composite, normalized via

$\hat{A}_{i j}=A_{i j} \frac{1-v^{2}}{2 H E_{1}} \quad ; \hat{B}_{i j}=B_{i j} \frac{1-v^{2}}{4 H^{2} E_{1}} \quad ; \hat{D}_{i j}=D_{i j} \frac{1-v^{2}}{8 H^{3} E_{1}}$,

\begin{tabular}{ccccc}
\hline \hline$N$ (Number of layers) & 20 & 200 & 2000 & $+\infty$ \\
\hline$\hat{A}_{11}$ & 0.5096 & 0.5224 & 0.5237 & 0.5239 \\
$\hat{A}_{22}$ & 0.5465 & 0.5261 & 0.5241 & 0.5239 \\
$\hat{A}_{12}$ & 0.1547 & 0.1584 & 0.1588 & 0.1589 \\
$\hat{A}_{66}$ & 0.1784 & 0.1821 & 0.1825 & 0.1825 \\
$\hat{B}_{16}$ & 0.0205 & 0.0225 & 0.0227 & 0.0227 \\
$\hat{B}_{26}$ & 0.0320 & 0.0356 & 0.0359 & 0.0359 \\
$\hat{D}_{11}$ & 0.0252 & 0.0260 & 0.0260 & 0.0261 \\
$\hat{D}_{22}$ & 0.0658 & 0.0636 & 0.0634 & 0.0634 \\
$\hat{D}_{12}$ & 0.0113 & 0.0121 & 0.0122 & 0.0122 \\
$\hat{D}_{66}$ & 0.0133 & 0.0140 & 0.0141 & 0.0141 \\
\hline \hline
\end{tabular}

so by understanding the behavior of the repetitive unit, the characteristics of the whole structure can be predicted. From experimental observation on samples from both species, the following results were found (Table I). The endocuticle is 3-4 times thicker than that of the exocuticle for Homarus americanus and 6-8 times thicker for Callinected sapidus [Figs. 2(a) and 3(a)]. A single $180^{\circ}$-stack in the endocuticle is $3-4$ times thicker than that of the exocuticle for both species [Figs. 2(c), 2(d), 3(c), and 3(d)]. The exocuticle has a fiber density higher than that of the endocuticle [Figs. 2(c), 2(d), 3(c), and $3(\mathrm{~d})]$. The endocuticle $180^{\circ}$-stack consists of more laminae than the exocuticle (Figs. 2 and 3 ).

Our model predicts that the higher number of laminae observed in the endocuticle will lead to a higher level of in-plane isotropy (Table III). It follows that the endocuticle, consisting of more laminae in each $180^{\circ}$-stack, could be closer to having an isotropic response than the exocuticle.

Also, the exocuticle has a higher fiber fraction than the endocuticle, which will result in a stronger and stiffer exocuticle because of the relatively high strength and stiffness of the chitin. Raabe et al. ${ }^{31}$ measured the hardness through nano-indentation of the Homarus americanus ${ }^{\prime}$ claw cuticle and showed a distinct decrease in hardness between the two regions. They reported an average hardness in the transverse direction $450 \mathrm{MPa}$ in the exocuticle, and $282 \mathrm{MPa}$ in the endocuticle. We believe that the change in fiber density of the two regions is an important reason for this hardness difference. [Exocuticle tanning (cross-liking between proteins) may also be a prominent factor for the hardness change, ${ }^{3}$ and will be left for future study.]

Finally, a qualitative comparison can be made between the Homarus americanus and the Callinected sapidus. The exoskeletons are constructed with the same materials and have similar morphology. However, the exoskeleton of the Homarus americanus claw is approximately 2-3 times thicker than the exoskeleton of the Callinected sapidus claw, and an $180^{\circ}$-stack of Homarus americanus is also approximately $2-3$ times thicker than that of $\mathrm{Cal}$ linected sapidus (Figs, 2 and 3). A $180^{\circ}$-stack in the Homarus americanus claw consists of more layers than in the Callinected sapidus claw (Figs, 2 and 3). Consequently, the claw exoskeleton from the Homarus americanus may be "more" isotropic than the Callinected sapidus claw.

\section{B. Pore canal fibers}

One of the most interesting features in the exoskeleton is the through-the-thickness pore canal system, consisting primarily of pore canals and pore canal fibers. Together with the layered structure, the three-dimensional 
structure of the exoskeleton is formed during molting ${ }^{16}$ and in-plane holes for the pore canal fibers are formed by the slightly departing and reuniting of chitin-protein macrofibrils in each plane (Fig. 6). The function of the pore canal system is to transport material during intermolting. ${ }^{16}$ In this work, we propose that the pore canal fibers also have the additional function of providing a mechanical reinforcement to the exoskeleton. To our knowledge, the latter has not been investigated.

Any plate or shell structure (e.g., an exoskeleton) that is subjected to an external transverse load (a load perpendicular to the surface) must be able to absorb shear stress and shear deformations. This requires a high interlaminar shear strength and shear stiffness, which are not automatically obtained in layered composite structures. In man-made materials, this is mitigated by stitching and in the investigated exoskeletons, it appears to be done by the pore canal fibers. A crustacean exoskeleton is frequently subjected to transverse load. For example, cracking another crustacean for a meal requires the claw to absorb significant amount of load. Furthermore, for the exoskeleton to withstand attacks from enemies, it must withstand bites and pinches that impose transverse shear. $^{32}$

We will discuss two possible mechanical functions of the pore canal fibers: increasing the transverse stiffness and increasing the interlaminar strength.

\section{Transverse stiffness}

In classic beam and plate theory (discussed in Sec. III. A), the effect of transverse shear is ignored. However, transverse shear can be an important factor for the overall mechanical behavior of thick laminates such as the exoskeletons considered here. The transverse shear can be incorporated from the relationship between the transverse shear forces $Q_{x}$ and $Q_{y}$ (Fig. 10) and the transverse shear strains $\epsilon_{x z}$ and $\epsilon_{y z}$ with the constitutive relationship $^{24}$ :

$$
\left[\begin{array}{l}
Q_{y} \\
Q_{x}
\end{array}\right]=2\left[\begin{array}{ll}
A_{44} & A_{45} \\
A_{45} & A_{55}
\end{array}\right]\left[\begin{array}{l}
\epsilon_{y z} \\
\epsilon_{x z}
\end{array}\right] \text {, }
$$

where

$$
\begin{aligned}
& A_{i j}=\frac{5}{4} \sum_{k=1}^{N}\left(\bar{Q}_{i j}\right)_{\mathrm{k}}\left[h_{\mathrm{k}}-h_{\mathrm{k}-1}-\frac{4}{3}\left(h_{\mathrm{k}}^{3}-h_{\mathrm{k}-1}^{3}\right) \frac{1}{H^{2}}\right], \\
& (i, j=4,5) \text {, }
\end{aligned}
$$

and $2 H$ is the total thickness of the laminate. $A_{i j}$ are the extensional stiffnesses and $\bar{Q}_{j}$ are the transformed stiffnesses, defined in the Appendix.

We believe that the pore canal fibers, by increasing the transverse shear moduli $\left(G_{13}\right.$ and $\left.G_{23}\right)$, will increase the transverse stiffness, resulting in smaller deformation during loading. To illustrate this, a $180^{\circ}$-stack (a unit cell) laminated plate (as modeled in previous section) with dimensions $\mathrm{a} \times b \times 2 \mathrm{H}$ is studied. All four edges are simply supported. The surface of the plate is loaded with a uniformly distributed pressure $q$. The necessary equations to solve this problem are summarized in the Appendix. The bio-material properties were previously derived (Table II), and the dimensions are estimated based on SEM images, listed in Table IV.

The normalized, maximum deflections $\hat{\omega}$ at the center of the plate are calculated using first-order shear deformation theory (Appendix) for various transverse shear moduli. Based on an analytical solution by Reddy ${ }^{25}$ and assuming transverse shear moduli $G_{23}=G_{13}$, our results (Fig. 11) show that higher transverse shear modulus corresponds to smaller maximum deflection. Thus, optimizing the appropriate properties of the pore canal fiber (e.g., increasing fiber density and diameter, increasing fiber-matrix friction) will increase the structure's ability of resisting transverse deformation from external force.

In-plane views of the endocuticle from both Homarus americanus and Callinected sapidus suggest that the pore canal fiber density is higher in Callinected sapidus than in Homarus americanus claw. However, the pore canal fiber diameter is larger in Homarus americanus than in Callinected sapidus [Table I and Figs. 6(b) and 6(d)]. Each equipped with an advantageous parameter over the other, the comparison of overall properties between the two species requires additional studies. This includes mechanical testing, such as nanoindentation, and will be conducted in a future study.

\section{Interlaminar strength}

In man-made laminates, transverse fibers running perpendicular to the laminates are used to increase the interlaminar strength. During interlaminar failure, debonding between the laminae is delayed by the stretching and the friction of the transverse fibers, resulting in absorption of more energy (e.g., Refs. 33 and 34). We believe that the pore canal system provides a similar naturedesigned strategy for preventing delamination. To illustrate this concept, we adopted the work by Jain and $\mathrm{Mai}^{35}$ where overall mode I failure under tensile load (Fig. 12) of stitched composite materials was investigated. Similar analyses can be done for overall mode II loading (inplane shear $)^{36}$ but are omitted for brevity.

The pore canal fibers in the exoskeleton are interpreted as the stitches in the Jain and Mai model (Fig. 12). ${ }^{35} \mathrm{We}$ assume that the pore canal fibers are attached to the

TABLE IV. Geometries, mechanical properties and external load for model of transverse stiffness.

\begin{tabular}{ccccc}
\hline \hline$a(\mu \mathrm{m})$ & $b(\mu \mathrm{m})$ & $2 H(\mu \mathrm{m})$ & $N$ & $Q\left(\mathrm{~N} / \mathrm{m}^{2}\right)$ \\
\hline 150 & 150 & 30 & 200 & 1 \\
\hline \hline
\end{tabular}




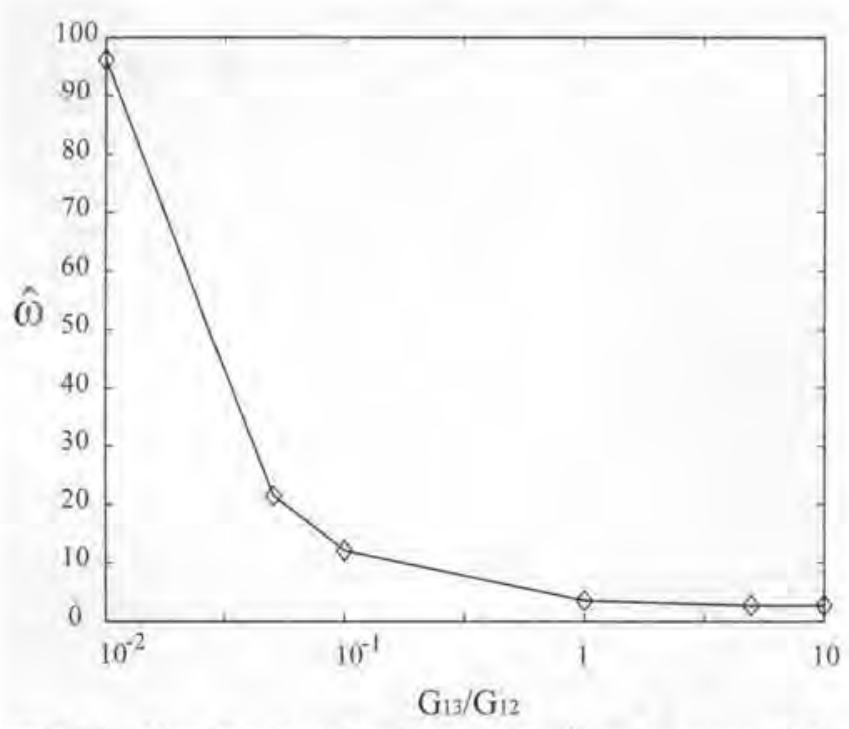

FIG, 11. Normalized maximum deflection ( $\hat{\omega})$ at the center of the plate against the transverse shear modulus $\left(G_{23}=G_{13}\right)$.

epicuticle at the top and to the structure below the exoskeleton. ${ }^{14,16}$ Thus, we can adapt the case of "interconnected-stitches" without "pre-tension" presented by Jain and Mai. ${ }^{35}$ For simplicity, we will refer to the pore canal fibers as "fibers" and the overall layered structure as the "matrix." Furthermore, we assume, again for simplicity, that the delamination occurs at the center of the structure and that the structure has the total thickness $2 H$. Failure will occur in several stages (assuming the fiber does not rupture in advance), as the external load $P$ increases (Fig. 13). First, the layered structure starts to delaminate between two layers. Then the fibers stretch between the delaminated surfaces and start to debond from the matrix [the debonded length is $0<Y<H$, Fig. 13(a)]. The debonded region reaches the thickness of the laminate, $Y=H$, where the force at the end of fiber $F_{0}$ emerges and increases with increasing loading [Figs. 13(b) and 13(c)]. Finally, the force in the fiber reaches the ultimate strength of the fiber $\sigma_{f u}$, causing the fiber to break.

During delamination, the debonded areas along the fiber provide frictional slip. This, along with the load absorbed by the fiber, contributes as a resistance force against crack propagation. The following equations describe the failure evolution and are directly from Jain and Mai. ${ }^{35}$

The crack opening displacement $\delta$ is related to the length of the crack bridged by stitches $\Delta a$ and determined based on the Euler beam equation with the governing beam equation:

$$
\begin{gathered}
E I \frac{d^{4} \delta}{d t^{4}}=-2 p(t), \\
0<t<\Delta a,
\end{gathered}
$$

where $E I$ is equivalent to $b D_{11}$ from the laminate theory, the coordinate $t$ originates at the crack tip (Fig. 12), and $p(t)$ is the distributed load from the closure forces given by

$$
p(t)=S_{D} \tau \pi d_{\mathrm{f}}\left[Y(t) U_{\mathrm{t}}+\left(H+\frac{F_{0}}{\tau \pi d_{\mathrm{f}}}\right)\left(1-U_{1}\right) U_{2}\right] .
$$

where $S_{D}$ is the fiber density, $\tau$ is the matrix-fiber interface shear stress ("friction stress"), and $d_{\mathrm{f}}$ is the (pore canal) fiber diameter (for simplicity, the fiber is modeled with circular cross-section). Furthermore, $U_{1}$ and $U_{2}$ are Heaviside step functions defined in the Appendix. The associate boundary conditions to the governing differential Eq. (7) are

$$
\begin{aligned}
\delta(0) & =0, \\
\frac{\mathrm{d} \delta}{\mathrm{d} t}(0) & =0, \\
\frac{\mathrm{d}^{2} \delta}{\mathrm{d}^{2}}(\Delta a) & =0, \\
\frac{\mathrm{d}^{3} \delta}{\mathrm{d} t^{3}}(\Delta a) & =-\frac{2 P}{E I} .
\end{aligned}
$$

Finally, the crack growth resistance can be expressed as

$$
\begin{aligned}
K_{\mathrm{R}}(\Delta a) & =K_{\mathrm{IC}}-K_{\mathrm{r}}(\Delta a) \\
& =K_{\mathrm{IC}}+\mathrm{C} \int_{i=0}^{\Delta a} p(t) \frac{1}{\sqrt{H}} f\left(\frac{t}{H}\right) \mathrm{d} t,
\end{aligned}
$$

where $K_{1 \mathrm{C}}$ is the fracture toughness of the laminate without the stitches ("intrinsic fracture toughness"), and $K_{\mathrm{r}}$ $(\Delta a)$ is the fracture toughness due to closure traction, with $f(t / H)$ being a dimensionless function defined in the Appendix.

We now use these equations developed for man-made composite materials to investigate the response of the exoskeletons. To highlight the effect the transverse fibers have on the fracture toughness, we focus on the change of crack growth resistance, $\Delta K_{\mathrm{R}}=-K_{\mathrm{r}}(\Delta a)$, due to the through-the-thickness pore canal fibers. The nominal parameters used are based on our sample observation and presented in Table $\mathrm{V}$. The interaction between the pore canal fibers and the matrix must be carefully considered in the model. Image analyses (Fig. 7) show that fibers in the exocuticle are well embedded in their surrounding matrix, which was also seen for Carcinus maenas (Green crab) by Compere. ${ }^{14}$ However, in the endocuticle, the pore canal fibers appear not to be in full contact with the surrounding matrix. Unfortunately, it is not possible from either our observation or available literature to establish 


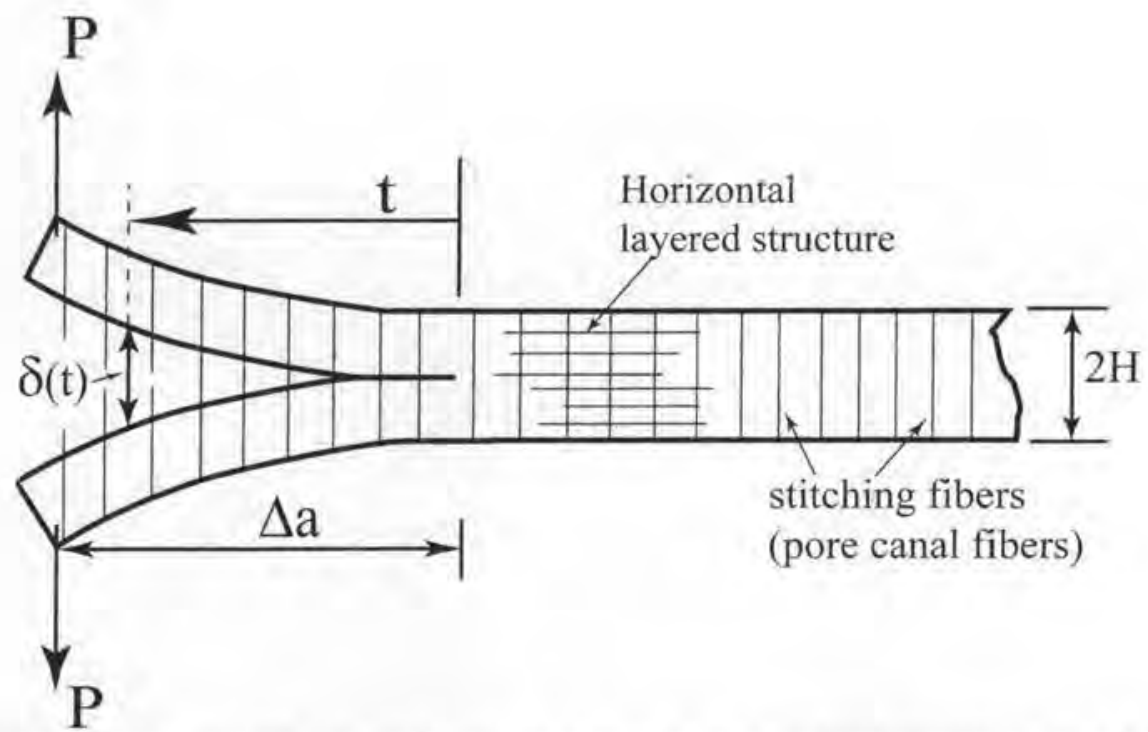

FIG. 12. Simplified model of double-cantilever laminated beam with through-the-thickness reinforcement, for mode I fracture. Beam thickness is $2 H$, bridge crack length is $\Delta a$, external force is $P, t$ is the distance from crack tip, and $\delta(t)$ is beam deflection at $t^{35}$

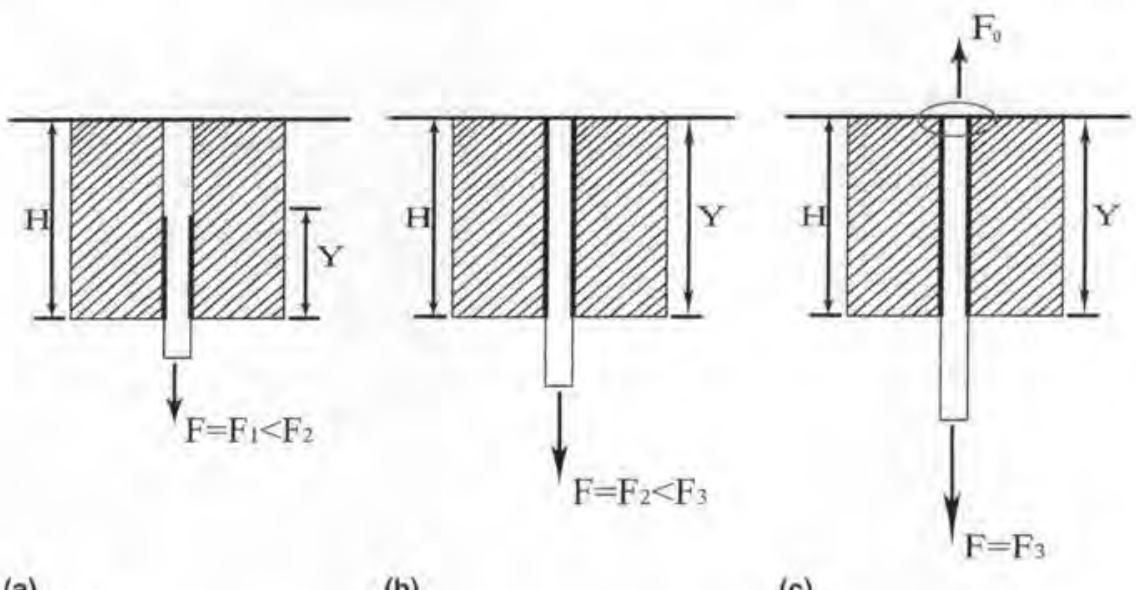

(a)

(b)

(c)

FIG. 13. Schematic illustration of evolving stages during the crack propagation: (a) initiation of debonding between fiber and matrix where $Y$ is the debonded length, (b) debonded region reaches the thickness of the beam, i.e., when $Y=H_{\text {, }}$ (c) $F_{0}$ emerges and increases until the fiber strength is reached. $F_{1}, F_{2}$, and $F_{3}$ are instantaneous fiber forces at (a), (b), and (c), respectively. ${ }^{35}$

TABLE V, Nominal properties used to investigate interlaminar strength.

\begin{tabular}{lc}
\hline \multicolumn{1}{c}{$H(\mu \mathrm{m})$} & 15 \\
\hline$N($ Number of layers $)$ & 200 \\
$d_{f}(\mu \mathrm{m})$ & 0.5 \\
$S_{\mathrm{D}}\left(\mu \mathrm{m}^{-2}\right)$ & 0.25 \\
$E_{f}(\mathrm{GPa})$ & 20 \\
$\sigma_{\mathrm{fu}}(\mathrm{MPa})$ & 500 \\
$E I$ or $b D_{\mathrm{M}}\left(\mathrm{Nm}^{2}\right)$ & $63 \times 10^{-14}$ \\
$\tau(\mathrm{MPa})$ & 50 \\
$K_{\mathrm{IC}}\left(\mathrm{Pa} \mathrm{m}^{-1 / 2}\right)$ & $1.5 \times 10^{7}$ \\
\hline \hline
\end{tabular}

the extent of contact between the pore canal fibers and the matrix in the endocuticle. Therefore, to explore all possible conditions on the delamination resistance, the lower bound of vanishing frictional stress between the pore canal fibers and the matrix is included when evaluating the mechanical response.

The change of the crack growth resistance from $K_{\mathrm{IC}}$ due to the pore canal fibers $\Delta K_{\mathrm{R}}$ as a function of bridged crack length $\Delta a$ is calculated for a range of cases, based on Eq. (9), and is summarized in Figs. 14 and 15. Due to the development of the bridging zone of the pore canal fibers in the debonding wake $\Delta K_{\mathrm{R}}$ increases until it reaches a constant value due to saturation of bridging zone size. Saturation of the bridging zone size corresponds to the region where a "steady state" is obtained from of the balance between new transverse fibers developed for bridging and old bridging transverse fibers ruptured. Selected parameters are varied in the simulations to demonstrate their influences on the interlaminar strength. The parameters are in some cases varied beyond 


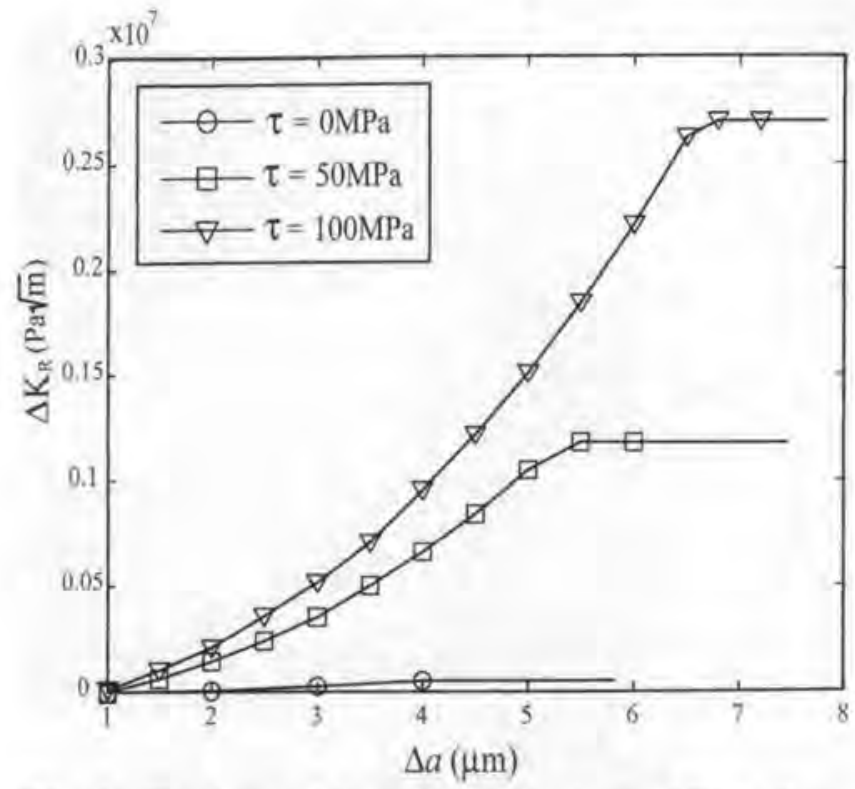

FIG. 15. The change of the crack growth resistance $\Delta K_{R}$ as a function of the bridged crack length $\Delta a$ and the influence due to the matrix-fiber interface shear stress $\tau$.

between the pore canal fiber and the matrix is neglected, although to a much lower extent (Fig. 15). Due to the absence of friction between the pore canal fiber and the matrix, only the forces at the embedded ends of the pore canal fibers contribute to the resistance against crack propagation, which explains the overall reduction on the improvement of crack growth resistance. However, if the pore canal fiber strength is high enough, the forces from the embedded ends of the pore canal fibers are able to compensate for the missing fiber-matrix frictional force. Then relatively large improvement on the interlaminar strength can be achieved (not shown for brevity). In exoskeletons, the model with frictional stress between the pore canal fibers and matrix is reasonable for exocuticles, whereas in endocuticles, the contribution from fibermatrix friction is low or none. However, in both cases, our proposed model suggests an improved interlaminar strength due to the presence of the pore canal fibers, with different degrees of enhancement.

In summary, the through-the-thickness pore canal fibers in the exoskeleton laminate improve the structure's resistance to mode I failure. In a similar manner, one can show that the mode II resistance is also increased due to the transverse pore canal fibers. Thus, the pore canal fibers have the important function of preventing the exoskeleton from splitting when subjected to external loads, such as an enemy attack.

\section{CONCLUDING REMARKS}

We have investigated exoskeletons from two common crustaceans: Homarus americanus (American Lobster) and Callinectes sapidus (Atlantic blue crab) with the aim of establishing their mechanical responses. The investigation was based on extensive SEM analysis to establish the morphology, followed by simple mechanics-based analyses to illustrate the mechanical responses.

In the study, we focused on the exoskeletons from the claws, since these structures are optimized for both light weight and strength, which are desired properties for many engineering applications. In both cases, the exoskeletons consist of three major regions: epicuticle, exocuticle, and endocuticle. The exocuticle and endocuticle are the major load-bearing structures and consequently the focus of this study. The endocuticle is about 6-8 times thicker than the exocuticle in the Callinectes sapidus and 3-4 times thicker in the Homarus americanus. However, the general characteristics of the two major regions are the same. They both consist of multiple fibrous layers that are oriented parallel to the external surface. Each layer consists of macrofibrils $(-30 \mathrm{~nm}$ in diameter) held together by proteins and calcified minerals. These layers are stacked with each layer rotating with a small angle relative to its neighboring layers. Our study supports the suggestion of the so-called Bouligand structure. Furthermore, pore canals and pore canal fibers are found to run perpendicular to these layers. The pore canal fibers are uniform in shape and extend straight through the layered structure. There is no evidence from our observation that they connect to or intersect each other.

From the established exoskeleton microstructure, we developed idealized models to analyze the overall mechanical response of the structural representative unit cell of the exoskeleton, which consists of layers forming an accumulated $180^{\circ}$ rotation of the fiber orientation. The $180^{\circ}$-stack is a regular antisymmetric laminate structure, which has a coupling between extension and bending (i.e., when loaded in pure tension, the laminate will twist or bend). Each $180^{\circ}$-stack consists of approximately 200 fibrous layers. Our mechanics-based analysis suggests this result in an almost isotropic response. Also our model shows that the presence of the transverse pore canals fibers has a significant influence on enhancing the transverse stiffness and strengthening the fracture resistance of the exoskeleton.

In summary, we have elucidated both the microscopic morphology and overall mechanical behavior of the exoskeleton for two common crustaceans, Homarus americanus and Callinectes sapidus. These investigations not only significantly expand the understanding of the structures, but also serve as foundations and inspirations for man-made composite structures.

\section{ACKNOWLEDGMENTS}

The authors would like to thank Dr. Kirk Czymmek and Ms. Shannon Modla at the Delaware Biotechnology 
Institute for helping in the sample preparations and SEM observations. The authors gratefully acknowledge support for this research from the University of Delaware Research Foundation (A.M.K. and L.C.) and grants from NIH (Nos. R01AR054385 and P20RR016458, L.W.).

\section{REFERENCES}

I. S. Weiner and L. Addadi: Design strategies in mineralized biological materials. J.Mater. Chem. 7, 689 (1997).

2. J.F.V. Vincent: Deconstructing the design of a biological material. J. Theor. Biol. 236, 73 (2005).

3. A.C. Neville: Biology of the Arthropod Cuticle (Springer-Verlag, New York, 1975).

4. K.M. Rudall and W. Kenching: Chitin system. Biol. Rev, Camb. Philos. Soc. 48, 597 (1973).

5. A.C. Neville, D.A.D. Parry, and J. Woodheadgalloway: Chitin crystallite in arthropod cuticle. J. Cell Sci. 21, 73 (1976).

6. R.Z. Wang, Z Suo, A.G. Evans, N. Yao, and I.A. Aksay: Deformation mechanisms in nacre. J. Mater. Res. 16, 2485 (2001).

7. A.G. Evans, Z. Suo, R.Z. Wang, I.A. Aksay, M.Y. He, and J.W. Hutchinson: Model for the robust mechanical behavior of nacre. J. Mater. Res. 16, 2475 (2001).

8. D.R. Katti, K.S. Katti, J.M. Sopp, and M. Sarikaya: 3D finite element modeling of mechanical response in nacre-based hybrid nanocomposites. Comput. Theor. Polym. Sci. 11, 397 (2001).

9. K. Katti, D.R. Katti, J. Tang, S. Pradhan, and M. Sarikaya: Modeling mechanical responses in a laminated biocomposite, Part II. Nonlinear responses and nuances of nanostructure. J. Mater. Sci. 40, 1749 (2005).

10. C. Sanchez, H. Arribart, and M.M.G. Guille: Biomimetism and bioinspiration as tools for the design of innovative materials and systems. Nat. Mater. 4, 277 (2005).

11. B.H. Ji and H.J. Gao: Mechanical properties of nanostructure of biological materials. J. Mech. Phys. Solids 52, 1963 (2004).

12. Y. Bouligan: Twisted fibrous arrangements in biological-materials and cholesteric mesophases. Tissue Cell 4, 189 (1972).

13. M.M. Girand-Guille: Plywood structure in nature. Curn Opin. Solid State Mater. Sci. 3, 221 (1998).

14. P. Compere and G. Goffinet: Ultrastructural shape and 3-dimensional organization of the intracuticular canal systems in the mineralized cuticle of the green crab Carcinus maenas. Tissue Cell 19, 839 (1987)

15. D. Raabe, P. Romano, C. Sachs, H. Fabritius, A. Al-Sawalmih. S. Yi, G. Servos, and H. Hartwig: Microstructure and crystallographic texture of the chitin-protein network in the biological composite material of the exoskeleton of the lobster Homarus americanus. Mater. Sci. Eng., A 421, 143 (2006).

16. P. Compere and G. Goffinet: Elaboration and ultrastructuralchanges in the pore canal system of the mineralized cuticle of Carcinus Maenas during the molting cycle. Tissue Cell 19, 859 (1987).

17, H. Mutvei: SEM studies on arthropod exoskeletons. Part I: Decapod crustaceans, Homarus gammarus L and Carcinus maenas (L.). Bull. Geol. Inst. Univ. Uppsala: New Series, 4(5), 73 (1974).

18. D. Raabe, C. Sachs, and P. Romano: The crustacean exoskeleton as an example of a structurally and mechamically graded biological nanocomposite material. Acta Mater. 53, 4281 (2005).

19. J.E. Hillerton and J.F.V. Vincent: Consideration of the importance of hydrophobic interactions in stabilizing insect cuticle. Int. J. Biol. Macromol, 5, 163 (1983).

20. J.E. Rebers and J,H. Willis: A conserved domain in arthropod cuticular proteins binds chitin Insect Biochem. Mol. Biol. 31, 1083 (2001).
21. U, Akiva, H.D. Wagner, and S. Weiner: Modelling the threedimensional elastic constants of parallel-fibred and lamellar bone. J. Mater. Sci. 33, 1497 (1998).

22. A. Fritsch, L. Dormieux, C. Hellmich, and J. Sanahuja: Micromechanics of crystal interfaces in polycrystalline solid phases of porous media: Fundamentals and application to strength of hydroxyapatite biomaterials. J. Mater. Sci. 42, 8824 (2007).

23. A. Fritsch and C. Hellmich: "Universal" microstructural patterns in cortical and trabecular, extracellular and extravascular bone materials: Micromechanics-based prediction of anisotropic elasticity. J. Theor. Biol: 244, 597 (2007).

24. J.R. Vinson and R.L. Sierakowski: The Behavior of Structures Composed of Composite Materials (Kluwer Academic Publishers, Boston, 2002)

25. J.N. Reddy: Mechanics of Laminated Composite Plates: Theory and Analysis (CRC Press, New York, 1997).

26. U.G.K. Wegst and M.F. Ashby: The mechanical efficiency of natural materials. Philos. Mag, 84, 2167 (2004).

27. J.D. Currey, A. Nash, and W. Bonfield: Calcified cuticle in the stomatopod smashing limb. J. Mater. Sci. 17, 1939 (1982).

28. R.F. Ker: Some structural and mechanical properties of locust and beetle cuticle. D. Phil. Thesis, University of Oxford (Oxford, UK, 1977).

29. J.F.V. Vincent: Insect cuticle: A paradigm for natural composites, in Symposia of the society for experimental biology (Cambridge University Press, Cambridge, UK, 1980).

30. J.F.V. Vincent: Arthropod cuticle: A natural composite shell system. Compos. A Appl. Sci. Manuf. 33, 1311 (2002).

31. C. Sachs, H. Fabritius, and D. Raabe: Hardness and elastic properties of dehydrated cuticle from the lobster Homarus anericanus obtained by nanoindentation. J. Mater. Res. 21, 1987 (2006).

32. D.E. Barshaw, K.L. Lavalli, and E. Spanier: Offense versus defense: Responses of three morphological types of lobsters to predation. Mar. Ecol, Prog. Ser. 256, 171 (2003).

33. K.A. Dransfield, L.K. Jain, and Y.W. Mai: On the effects of stitching in CFRPs I. Mode I delamination toughness. Compos. Sci. Technol. 58, 815 (1998).

34. L.K. Jain, K.A. Dransfield, and Y.W. Mai: On the effects of stitching in CFRPs II. Mode II delamination toughness. Compos. Sci. Technol. 58, 829 (1998).

35. L. Jain and Y. Mai: On the effect of stitching on mode-I delamination toughness of laminated composites. Compos. Sci. Technol. 51, 331 (1994).

36. L.K. Jain and Y.W. Mai: Analysis of stitched laminated enf specimens for interlaminar mode-II fracture-toughness. Int. J. Fract. 68, 219 (1994).

37. H.T, Hahn: Simplified formulas for elastic moduli of unidirectional continuous fiber composites. Compos. Technol. Rev. 2, 5 (1980).

\section{APPENDIX}

\section{A. Classic laminate theory}

The resultant in-plane normal forces $N_{x}$ and $N_{y}$ and in-plane shear force $N_{x y}$, as well as the bending moments on the surfaces with normals in the $x$ and $y$ direction $M_{x}$ and $M_{y}$, and the twist of the laminate $M_{x y}$ can be related to the normal strains $\epsilon_{x}^{0}$, $\epsilon_{y}^{0}$ and shear strain $\gamma_{x y}^{0}$, all at the mid-plane, and the curvatures $K_{x}, K_{\mathrm{y}}$ and $K_{x y}$ according to $^{24.25}$ (Fig. 10)

$$
\mathrm{N}=\mathrm{A} \epsilon+\mathrm{B} \kappa, \quad \mathrm{M}=\mathrm{B} \epsilon+\mathrm{D} \kappa .
$$


with

$$
\begin{aligned}
& \mathbf{N}=\left[\begin{array}{c}
N_{x} \\
N_{y} \\
N_{x y}
\end{array}\right], \quad \mathbf{M}=\left[\begin{array}{c}
M_{x} \\
M_{y} \\
M_{x y}
\end{array}\right], \quad \boldsymbol{\epsilon}=\left[\begin{array}{c}
\epsilon_{x}^{0} \\
\epsilon_{y}^{0} \\
\gamma_{x y}^{0}
\end{array}\right], \\
& \mathbf{\kappa}=\left[\begin{array}{c}
\kappa_{x} \\
\kappa_{y} \\
\kappa_{x y}
\end{array}\right], \quad \mathbf{A}=\left[\begin{array}{ccc}
A_{11} & A_{12} & 0 \\
A_{12} & A_{22} & 0 \\
0 & 0 & A_{66}
\end{array}\right], \\
& \mathbf{B}=\left[\begin{array}{ccc}
0 & 0 & B_{16} \\
0 & 0 & B_{26} \\
B_{16} & B_{26} & 0
\end{array}\right], \quad \mathbf{D}=\left[\begin{array}{ccc}
D_{11} & D_{12} & 0 \\
D_{12} & D_{22} & 0 \\
0 & 0 & D_{66}
\end{array}\right],
\end{aligned}
$$

where $A_{i j}$ are extensional stiffnesses and $B_{i j}$ are bendingextension coupling stiffnesses, and $D_{i j}$ are flexural stiffnesses given by

$$
\begin{aligned}
& A_{i j}=\sum_{k=1}^{N}\left(\bar{Q}_{i j}\right)_{k}\left(h_{k}-h_{k-1}\right), \\
& B_{i j}=\frac{1}{2} \sum_{k=1}^{N}\left(\bar{Q}_{i j}\right)_{k}\left(h_{k}^{2}-h_{k-1}^{2}\right), \\
& D_{i j}=\frac{1}{3} \sum_{k=1}^{N}\left(\bar{Q}_{i j}\right)_{k}\left(h_{k}^{3}-h_{k-1}^{3}\right),
\end{aligned}
$$

where $h_{k}$ is the distance from mid-plane to the upper side of the $k$ th layer and $h_{k-1}$ is the distance to the lower side of the $k$ th layer. $h$ is 0 on mid-plane and negative below the mid-plane. The layers are numbered from 1 to $N$ from the bottom to the top of the laminate [Fig. 10(a)]. Furthermore, $\bar{Q}_{i j}$ are the transformed stiffnesses

$$
\begin{aligned}
\bar{Q}_{11}= & Q_{11} \cos ^{4} \theta+2\left(Q_{12}+2 Q_{66}\right) \sin ^{2} \theta \cos ^{2} \theta \\
& +Q_{22} \sin ^{4} \theta, \\
\bar{Q}_{12}= & \left(Q_{11}+Q_{22}-4 Q_{66}\right) \sin ^{2} \theta \cos ^{2} \theta \\
& +Q_{12}\left(\sin ^{4} \theta+\cos ^{4} \theta\right), \\
\bar{Q}_{22}= & Q_{11} \sin ^{4} \theta+2\left(Q_{12}+2 Q_{66}\right) \sin ^{2} \theta \cos ^{2} \theta \\
& +Q_{22} \cos ^{4} \theta, \\
\bar{Q}_{16}= & \left(Q_{11}-Q_{12}-2 Q_{66}\right) \sin \theta \cos ^{3} \theta+\left(Q_{12}\right. \\
& \left.-Q_{22}+2 Q_{66}\right) \sin ^{3} \theta \cos \theta, \\
\bar{Q}_{26}= & \left(Q_{11}-Q_{12}-2 Q_{66}\right) \sin ^{3} \theta \cos \theta+\left(Q_{12}\right. \\
& \left.-Q_{22}+2 Q_{66}\right) \sin \theta \cos ^{3} \theta, \\
\bar{Q}_{66}= & \left(Q_{11}+Q_{22}-Q_{12}-2 Q_{66}\right) \sin ^{2} \theta \cos ^{2} \theta \\
& +Q_{66}\left(\sin ^{4} \theta+\cos ^{4} \theta\right),
\end{aligned}
$$

with the reduced stiffnesses:

$$
\begin{aligned}
& Q_{11}=\frac{E_{1}}{1-v_{12} \nu_{21}}, \\
& Q_{22}=\frac{E_{2}}{1-v_{12} v_{21}}, \\
& Q_{12}=\frac{v_{21} E_{1}}{1-v_{12} v_{21}}=\frac{v_{12} E_{2}}{1-v_{12} v_{21}}, \\
& Q_{66}=G_{12},
\end{aligned}
$$

where we note that $v_{12} E_{2}=v_{21} E_{1}$.

\section{B. Hahn's equations}

Equations for obtaining the elastic properties of a composite based on the fiber and matrix materials are summarized here. ${ }^{24,37}$ These equations are based on circular cross-section fibers, which are randomly distributed unidirectionally in the matrix.

The elastic constants $E_{11}, v_{12}, G_{12}, G_{23}, K_{\mathrm{T}}$ (the plane strain bulk modulus) can be expressed in the generalized functional form of

$$
P=\frac{P_{\mathrm{f}} V_{\mathrm{f}}+\eta P_{\mathrm{m}} V_{\mathrm{m}}}{V_{\mathrm{f}}+\eta V_{\mathrm{m}}},
$$

where $P$ is specific elastic constant and subscripts $f$ and $\mathrm{m}$ denote fiber and matrix, respectively. $V_{\mathrm{f}}$ and $V_{\mathrm{m}}$ are volume fractions of the fibers and matrix, respectively. $P_{f}, P_{\mathrm{m}}$, and $\eta$ are shown in Table A1. In Table A1 the following parameters are used:

$$
\begin{aligned}
\eta_{6} & =\frac{1+G_{\mathrm{m}} / G_{12 \mathrm{f}}}{2}, \\
\eta_{4} & =\frac{3-4 v_{\mathrm{m}}+G_{\mathrm{m}} / G_{23 \mathrm{f}}}{4\left(1-v_{\mathrm{m}}\right)}, \\
\eta_{\mathrm{K}} & =\frac{1+G_{\mathrm{m}} / K_{\mathrm{f}}}{2\left(1-v_{\mathrm{m}}\right)}, \\
K_{\mathrm{f}} & =\frac{E_{\mathrm{f}}}{2\left(1-v_{\mathrm{f}}\right)}, \\
K_{\mathrm{m}} & =\frac{E_{\mathrm{m}}}{2\left(1-v_{\mathrm{m}}\right)},
\end{aligned}
$$

and for an isotropic matrix

$$
G_{\mathrm{m}}=E_{\mathrm{m}} / 2\left(1+v_{\mathrm{m}}\right)
$$

TABLE A1. Elastic constants based on Hahn's equation.

\begin{tabular}{cllll}
\hline \hline $\begin{array}{c}\text { Elastic } \\
\text { constant }\end{array}$ & $P$ & $P_{\mathrm{f}}$ & $P_{\mathrm{m}}$ & $\eta$ \\
\hline$E_{11}$ & $E_{11}$ & $E_{\mathrm{f}}$ & $E_{15}$ & 1 \\
$\nu_{12}$ & $v_{12}$ & $y_{127}$ & $\nu_{\mathrm{m}}$ & 1 \\
$G_{12}$ & $1 / G_{12}$ & $1 / G_{121}$ & $1 / G_{\mathrm{m}}$ & $\eta_{\sigma}$ \\
$G_{23}$ & $1 / G_{23}$ & $1 / G_{12 \mathrm{r}}$ & $1 / G_{\mathrm{m}}$ & $\eta_{A}$ \\
$K_{\mathrm{T}}$ & $1 / K_{\mathrm{T}}$ & $1 / K_{\mathrm{f}}$ & $1 / K_{\mathrm{m}}$ & $\eta_{\mathrm{f}}$ \\
\hline \hline
\end{tabular}


Then

$$
E_{22}=E_{33}=\frac{4 K_{\mathrm{T}} G_{23}}{K_{\mathrm{T}}+m G_{23}},
$$

where

$$
m=1+\frac{4 K_{\mathrm{T}} v_{12}^{2}}{E_{11}} .
$$

\section{Laminated plate with simply supported edges}

Here we summarize the Navier solution for rectangular laminated plate with simply supported boundary conditions at all four edges using first-order shear deformation theory. ${ }^{25}$ Assume the plate is $a$ wide in the $x$ direction and $b$ wide in the $y$ direction. A solution exists for an antisymmetric laminate. For this problem, the boundary conditions are satisfied with the expansions:

$$
\begin{aligned}
& u_{0}(x, y)=\sum_{n=1}^{\infty} \sum_{m=1}^{\infty} U_{\mathrm{mn}} \sin \alpha x \cos \beta y, \\
& v_{0}(x, y)=\sum_{n=1}^{\infty} \sum_{m=1}^{\infty} V_{\mathrm{mn}} \cos \alpha x \sin \beta y, \\
& \omega_{0}(x, y)=\sum_{n=1}^{\infty} \sum_{m=1}^{\infty} W_{\mathrm{mn}} \sin \alpha x \sin \beta y . \\
& \phi_{\mathrm{r}}(x, y)=\sum_{n=1}^{\infty} \sum_{m=1}^{\infty} X_{\mathrm{mn}} \cos \alpha x \sin \beta y . \\
& \phi_{\mathrm{y}}(x, y)=\sum_{n=1}^{\infty} \sum_{m=1}^{\infty} Y_{\mathrm{mn}} \sin \alpha x \cos \beta y .
\end{aligned}
$$

where $\alpha=m \pi / a, \beta=n \pi / b$ and $u_{0}, v_{0}$, and $\omega_{0}$ are displacements in the $x, y$, and $z$ directions, $\phi_{x}$ and $\phi_{y}$ are rotations about the $x$ and $y$ axes. Furthermore, the coefficients $U_{\mathrm{mn}}, U_{\mathrm{mn}}, W_{\mathrm{mn}}, X_{\mathrm{mn}}$, and $Y_{\mathrm{mn}}$ can be determined from

$$
\left[\begin{array}{ccccc}
\hat{s}_{11} & \hat{s}_{12} & 0 & \hat{s}_{14} & \hat{s}_{15} \\
\hat{s}_{12} & \hat{s}_{22} & 0 & \hat{s}_{24} & \hat{s}_{25} \\
0 & 0 & \hat{s}_{33} & \hat{s}_{34} & \hat{s}_{35} \\
\hat{s}_{14} & \hat{s}_{24} & \hat{s}_{34} & \hat{s}_{44} & \hat{s}_{45} \\
\hat{s}_{15} & \hat{s}_{25} & \hat{s}_{35} & \hat{s}_{45} & \hat{s}_{55}
\end{array}\right]\left[\begin{array}{c}
U_{\mathrm{mn}} \\
V_{\mathrm{mn}} \\
W_{\mathrm{mn}} \\
X_{\mathrm{mn}} \\
Y_{\mathrm{mn}}
\end{array}\right]=\left\{\begin{array}{c}
0 \\
0 \\
Q_{\mathrm{mn}} \\
0 \\
0
\end{array}\right\},
$$

where elements in the $5 \times 5$ matrix can be obtained based on stiffness matrices $A, B$, and $D$ as

$$
\begin{aligned}
& \hat{s}_{11}=\left(A_{11} \alpha^{2}+A_{66} \beta^{2}\right), \\
& \hat{s}_{12}=\left(A_{12}+A_{66}\right) \alpha \beta, \\
& \hat{s}_{13}=0, \\
& \hat{s}_{14}=2 B_{16} \alpha \beta, \\
& \hat{s}_{15}=\left(B_{16} \alpha^{2}+G_{26} \beta^{2}\right), \\
& \hat{s}_{22}=\left(A_{66} \alpha^{2}+A_{22} \beta^{2}\right), \\
& \hat{s}_{23}=0, \\
& \hat{s}_{24}=\hat{s}_{15}, \\
& \hat{s}_{25}=2 B_{26} \alpha \beta, \\
& \hat{s}_{33}=K\left(A_{55} \alpha^{2}+A_{44} \beta^{2}\right), \\
& \hat{s}_{34}=K A_{55} \alpha, \\
& \hat{s}_{35}=K A_{44} \beta, \\
& \hat{s}_{44}=\left(D_{11} \alpha^{2}+D_{66} \beta^{2}+K A_{55}\right), \\
& \hat{s}_{45}=\left(D_{12}+D_{66}\right) \alpha \beta, \\
& \hat{s}_{55}=\left(D_{66} \alpha^{2}+D_{22} \beta^{2}+K A_{44}\right),
\end{aligned}
$$

where $K$ is the shear correction coefficient, determined from the equivalence of the strain energy due to transverse shear stresses predicted with $K$ and the strain energy due to the true transverse stresses predicted by the three-dimensional elasticity theory.

$Q_{\mathrm{mn}}$ can be obtained from expansion of the distributed pressure $q$ according to

$$
q=\sum_{n=1}^{\infty} \sum_{m=1}^{\infty} Q_{n m} \sin \alpha x \cos \beta y .
$$

Finally, the normalized deflection is obtained according to

$$
\hat{\omega}=\left(\frac{E_{2} H^{3}}{b^{4} q}\right) \omega_{0},
$$

where $2 \mathrm{H}$ is the plate thickness and $q$ is the distributed pressure.

\section{Transverse fibers}

The parameters needed for determining the resistance that the transverse fibers add to the delamination strength of stitched fiber composites are summarized here. All equations are directly from Jain and Mai's work. ${ }^{35}$ Let $\tau$ be the matrix-fiber interface shear stress ("friction stress"), and $d_{\mathrm{f}}$ the fiber diameter. For $Y<H$ in Fig. 13, the force in the transverse fiber caused by frictional slip $F$ is given by the functional

$$
F[\delta(Y)]=\tau \pi d_{f} Y .
$$


where the crack opening displacement $\delta(Y)$ is given by

$$
\delta(Y)=\left[Y-\frac{H}{r} \ln \left(\frac{Y r}{H}+1\right)\right]\left[1+\frac{Y r}{H}\right],
$$

where $r$ is the extensibility ratio defined by

$$
r=\frac{\tau \pi d_{\mathrm{f}} H}{A_{\mathrm{f}} E_{\mathrm{f}}},
$$

where $A_{f}$ is the fiber cross-section area and $E_{f}$ is the elastic modulus of the fiber.

For $Y=H$, all additional force is absorbed by the fiber only (no additional absorption through friction). The force absorbed by the fiber is

$$
F_{0}=F-F_{\mu},
$$

where $F_{\mu}$ is the maximum force absorbed through friction given by

$$
F_{\mu}=\tau \pi d_{\mathrm{f}} H .
$$

Thus, once the fiber is completely debonded, Eq. (A17) is replaced by

$$
F\left[\delta\left(F_{\mathrm{o}}\right)\right]=\tau \pi d_{\mathrm{f}} H+F_{0},
$$

where

$$
\begin{aligned}
\delta\left(F_{0}\right)= & {\left[H-\frac{H}{r} \ln \left(\frac{r}{1+F_{0} /\left(A_{\mathrm{f}} E_{\mathrm{f}}\right)}+1\right)\right] } \\
& {\left[1+r+F_{\mathrm{o}} /\left(A_{\mathrm{f}} E_{\mathrm{f}}\right)\right] . }
\end{aligned}
$$

$U_{1}$ and $U_{2}$ are Heaviside step functions of the form

$$
\begin{aligned}
U_{1} & =U_{1}\left(\left[H-\frac{H}{r} \ln (1+r)\right](1+r)-\delta(t)\right), \\
U_{2} & =U_{2}\left[\sigma_{\mathrm{fu}} A_{\mathrm{f}}-\tau \pi d_{\mathrm{f}} H-F_{\mathrm{o}}(t)\right],
\end{aligned}
$$

and $\sigma_{\mathrm{fu}}$ is the fiber strength. We recall that a Heaviside step function is defined by

$$
U(x)=\left\{\begin{array}{l}
1, x>0 \\
0, x \leqslant 0
\end{array} .\right.
$$

The fracture toughness due to the closure traction $\mathrm{K}_{\mathrm{T}}(\Delta a)$ contains the dimensionless function $f(t / H)$, defined by

$$
\begin{aligned}
f\left(\frac{t}{H}\right)= & \sqrt{12}\left(\frac{t}{H}+0.673\right)+\sqrt{\frac{2 H}{\pi t}} \\
& -\left[0.815\left(\frac{t}{H}\right)^{0.619}+0.429\right]^{-1} .
\end{aligned}
$$

